\title{
The Topology of Real Algebraic Varieties
}

\author{
János Kollár
}

\section{Contents}

$\begin{array}{ll}\text { 1. Introduction } & 197\end{array}$

2. Minimal Models of Real Algebraic Surfaces 203

3. The Minimal Model Program for Real 3-folds 209

4. The Nash Conjecture for Nonprojective Threefolds 218

5. The Topology of Real Del Pezzo Surfaces 223

$\begin{array}{ll}\text { References } & 229\end{array}$

\section{Introduction}

The aim of these notes is to give a survey of some of the recent results and directions in real algebraic geometry. We are mainly interested in the topological aspects of the theory.

The study of real algebraic varieties is classical, it even predates algebraic geometry over other fields. Despite this, in the last 50 years real algebraic geometry developed to a large extent independently of general algebraic geometry, notwithstanding the efforts of a few people active in both fields. There are even two quite different definitions of what a "real algebraic variety" should be. (See (21) for my conventions which come from algebraic geometry.)

It seems to me that this separation has been detrimental to both disciplines. Real algebraic geometry can gain by utilizing the methods of complex algebraic geometry and algebraic geometry can connect with many other fields through real questions. As an example, let me just mention the theory of hyperbolic PDE-s which leads to very interesting problems about real algebraic hypersurfaces and their deformations.

1. In studying the toplogy of real algebraic varieties, two main directions emerge:

(1.1) (Recognition problem) Which properties of the set of real points can be determined from algebraic geometry over $\mathbb{C}$. The simplest example is the dimension. If $X$ is smooth then the real dimension of $X(\mathbb{R})$ (the set of real 
points) is the same as the complex dimension of $X(\mathbb{C})$ (the set of complex points), except that $X(\mathbb{R})$ may be empty.

(1.2) (Realization problem) Which topological data can be realized by real algebraic varieties. The answer here is especially interesting if the realization establishes a close connection between topology and algebra. Here the nicest example is the realization of semi-simple Lie groups by real algebraic matrix groups. Unfortunately, such close connection between topology and algebra is rather rare.

The first general result comparing topological properties of the real points with complex analytic properties is due to [Harnack 1876], who proved the following (cf. [Shafarevich72, VII.4]).

THEOREM 2. Let $B$ be a smooth, projective, real algebraic curve. Then $\#($ connected components of $B(\mathbb{R})) \leq g(B)+1$.

Note the important feature of this result that we only have an inequality. Namely, we assert that if the complex curve is simple than the topology of the real points is also simple. In general we can not expect any implication the other way. Indeed, the set of real solutions of $\left(x^{2 d}+y^{2 d}=1\right)$ is always a circle but the genus of the complex curve goes to infinity.

This leads to the following general question, which is the main topic of these notes.

Main Problem 3. Prove that the complexity of the set of real points $X(\mathbb{R})$ of a real algebraic variety can be bounded by invariants of the complex variety $X(\mathbb{C})$.

1.1. Topological Methods. The simplest and prettiest theorems of this type are purely topological. Complex conjugation is an involution on $X(\mathbb{C})$ whose fixed point set is $X(\mathbb{R})$. It was noticed by [Thom65] and [Sullivan71] that general properties of involutions give the following:

THEOREM 4. For any real algebraic variety $X$,

$$
\begin{aligned}
\sum_{i} h^{i}\left(X(\mathbb{R}), \mathbb{Z}_{2}\right) & \leq \sum_{i} h^{i}\left(X(\mathbb{C}), \mathbb{Z}_{2}\right), \quad \text { and } \\
\sum_{i} h^{i}\left(X(\mathbb{R}), \mathbb{Z}_{2}\right) & \equiv \sum_{i} h^{i}\left(X(\mathbb{C}), \mathbb{Z}_{2}\right) \bmod 2 .
\end{aligned}
$$

By considering the degree as a basic invariant, [Milnor64] proved the following bound.

Theorem 5. Let $X \subset \mathbb{P}^{n}$ be a real algebraic variety of degree $d$. Then

$$
\sum_{i} h^{i}\left(X(\mathbb{R}), \mathbb{Z}_{2}\right) \leq d(2 d-1)^{n-1}
$$

These results are quite strong in dimensions 1 and 2 , since in these dimensions the Betti numbers pretty much determine the topology. The situation drastically changes starting with dimension 3 , where homotopy theoretic information becomes indispensable.

A detailed survey of the homological study of real algebraic varieties is given in [Degtyarev-Kharlamov00].

It is also of interest to study other, more algebraic, invariants of $X$. One of the natural candidates is the Kodaira dimension of $X$, denoted by $\kappa(X)(22)$. 
There is no way to bound the sum of the Betti numbers in terms of the Kodaira dimension, but one may hope to read off certain properties of $X(\mathbb{R})$ from the Kodaira dimension. Here we concentrate on the simplest case of varieties with Kodaira dimension $-\infty$.

This question is interesting, and largely unsolved, even for complex varieties. Using basic properties of Gromov-Witten invariants, [Kollár98a, 4.2.10] proves that the case of negative Kodaira dimension is an essentially topological property of the set of complex points:

THEOREM 6. Let $X$ be a smooth, complex, projective variety. Then one can decide from the underlying symplectic manifold $(X(\mathbb{C}), \omega)$ whether $\kappa(X)=-\infty$ or not.

1.2. The Nash Conjecture. In the realization problem the first general result was proved in [Nash52] and later improved by [Tognoli73]. Their result says that every compact differentiable manifold is algebraic:

TheOREM 7. [Nash52, Tognoli73] Let $M^{n}$ be a compact differentiable manifold. Then there are real polynomials $f_{i}\left(x_{1}, \ldots, x_{N}\right)$ such that their common zero set

$$
X(\mathbb{R}):=\left\{\mathbf{x} \in \mathbb{R}^{N}: f_{i}(\mathbf{x})=0 \forall i\right\} \subset \mathbb{R}^{N}
$$

is smooth and diffeomorphic to $M^{n}$.

Nash then went on to ask if this result can be improved by specifying various algebraic properties of the variety $X$. From (5) we see that $X$ can not have bounded degree, in fact no bounded subset of algebraic varieties can represent all manifolds. Thus it is natural to ask if one can restrict the Kodaira dimension of $X$. It is easy to see that one can always make the canonical class of $X$ ample:

EXAMPLE 8 . Let $X \subset \mathbb{P}^{n}$ be a smooth real algebraic variety. Let $\left(x_{0}: \cdots: x_{n}\right)$ be coordinates on $\mathbb{P}^{n}$ and $(s: t)$ coordinates on $\mathbb{P}^{1}$. Let $Y \subset X \times \mathbb{P}^{1}$ be the hypersurface defined by the equation

$$
s^{3}\left(x_{0}^{2 d}+\cdots+x_{n}^{2 d}\right)=t^{3}\left(x_{0}^{2 d}+2 x_{0}^{2 d}+\cdots+(n+1) x_{n}^{2 d}\right) .
$$

Then $Y(\mathbb{R}) \rightarrow X(\mathbb{R})$ is a diffeomorphism and the canonical class of $Y$ is very ample for $d \gg 1$.

There are, however, no general constructions which produce a variety of lower Kodaira dimension starting with a variety of higher Kodaira dimension, while keeping the dimension unchanged. (For any $X, \kappa\left(X \times \mathbb{P}^{1}\right)=-\infty$, but this does not yield anything interesting topologically.) Thus it is of interest to see if one can realize every differentiable manifold with varieties of small Kodaira dimension. Nash pointed out that the following would be the strongest possible result:

CONJECTURE 9. [Nash52, p.421] Let $M^{n}$ be a compact, connected, differentiable manifold. Then there is a smooth real algebraic variety $X^{n}$ such that $X$ is birational to $\mathbb{P}^{n}$ and $X(\mathbb{R})$ is diffeomorphic to $M^{n}$.

This is obvious for curves. Unbeknownst to Nash, this question has been settled for surfaces much earlier. It is sign of the separation of various branches of mathematics that this seemed not to have been realized for quite a few years.

TheOREM 10. [Comessatti14] Let $S$ be a smooth real algebraic surface. Assume that $S$ is birational to $\mathbb{P}^{2}$ and $S(\mathbb{R})$ is orientable.

Then $S(\mathbb{R})$ is either a sphere or a torus. 
Several attempts have been made to use the result of [Comessatti14] to get higher dimensional counter examples, for instance for threefolds of the form $S^{1} \times$ (high genus surface) or for 4 -folds which are the product of 2 high genus surfaces. This type of trivial dimension increase frequently works in complex algebraic geometry but it does not seem to work in this example.

[Benedetti-Marin92] showed that for every 3-manifold $M^{3}$ there is a singular real algebraic variety $X$ birational to $\mathbb{P}^{3}$ such that $X(\mathbb{R})$ is homeomorphic to $M^{3}$. [Akbulut-King91] and [Mikhalkin97] show that the related "topological Nash conjecture" is true.

The 3-dimensional Nash conjecture has been settled recently in a series of papers [Kollár98b, Kollár99a, Kollár99b, Kollár00a, Kollár00b]. The first 4 papers provide a negative solution and the last one a positive solution. It turns out that the seemingly innocuous projectivity assumption drastically changes the nature of the problem. This is a completely new phenomenon of 3 -fold geometry. The precise statements are the following. (The relevant concepts of 3-manifolds topology are recalled in (17).)

THEOREM 11 (The Nash conjecture fails in dimension 3).

Let $X$ be a smooth, real, projective 3-fold birational to $\mathbb{P}^{3}$. Assume that $X(\mathbb{R})$ is orientable. Then every connected component of $X(\mathbb{R})$ is among the following:

1. Seifert fibered,

2. connected sum of lens spaces,

3. torus bundle over $S^{1}$ or doubly covered by a torus bundle over $S^{1}$,

4. finitely many other possible exceptions, or

5. obtained from one of the above 3-manifolds by repeatedly taking connected sum with $\mathbb{R P}^{3}$ and $S^{1} \times S^{2}$.

THEOREM 12 (The Nash conjecture holds in dimension 3).

For every compact, connected, differentiable 3-manifold $M$ there is a compact complex manifold $X$ which can be obtained from $\mathbb{P}^{3}$ by a sequence of smooth, real blow ups and downs such that $M$ is diffeomorphic to $X(\mathbb{R})$.

The two statements are not in contradiction since blowing down is not always possible among projective varieties. Thus we have to work in the slightly larger category of Moishezon manifolds or algebraic spaces, see section 4.

REMARK 13. The assumptions of (11) can be weakened considerably. Namely, (11) also holds if we assume the following much weaker conditions:

1. $X$ has Kodaira dimension $-\infty$ (instead of assuming $X$ to be birational to $\left.\mathbb{P}^{3}\right)$

2. $X(\mathbb{R})$ does not contain any of the following types of embedded surfaces:

(a) 2-sided $\mathbb{R P}^{2}$;

(b) 1-sided torus;

(c) 1-sided Klein bottle with nonorientable neighborhood,

(instead of assuming $X(\mathbb{R})$ to be orientable). In this case $X(\mathbb{R})$ may have several connected components and each satisfies the conclusions of (11). I do not know what happens if some components of $X(\mathbb{R})$ are orientable and some are not.

The proof of (11) establishes a close connection between certain algebraic properties of $X(\mathbb{C})$ and geometric structures of $X(\mathbb{R})$. In one case, which is known to contain only finitely many types of varieties, such a connection has not been proved, 
and this accounts for the finitely many unknown cases. I believe, however, that there are no exceptions:

Conjecture 14. The cases (11.3-4) do not occur. (To be precise, if a torus bundle occurs then it is also Seifert fibered.)

It is also of interest to note that the conditions (13.2.a-c) hold for every hyperbolic 3-manifold. Thus we obtain the following. (Again I conjecture that there are no exceptions.)

THEOREM 15. There are only finiteley many hyperbolic 3-manifolds (orientable or not) among the $X(\mathbb{R})$ where $X$ is a smooth, projective, real algebraic 3-fold with Kodaira dimension $-\infty$.

A similar result was obtained in all dimensions by Viterbo, using stronger conditions on rational curves. (See [Kharlamov00] for a discussion of this and related topics.)

THEOREM 16. [Viterbo98] Let $X$ be a smooth, projective, real algebraic variety of dimension $n \geq 3$. Assume that $H_{2}(X(\mathbb{C}), \mathbb{Z}) \cong \mathbb{Z}$ and that $X(\mathbb{C})$ is covered by rational curves $C_{\lambda}$ such that $\left[C_{\lambda}\right] \in H_{2}(X(\mathbb{C}), \mathbb{Z})$ is a generator.

Then $X(\mathbb{R})$ does not carry any metric with negative sectional curvature.

DEFINITION 17. For relatively prime $0<q<p$ consider the action of $\mathbb{Z}_{p}$ on $S^{3} \sim\left(\left|x^{2}\right|+\left|y^{2}\right|=1\right) \subset \mathbb{C}^{2}$ given by $(x, y) \mapsto\left(e^{2 \pi i / p} x, e^{2 \pi i q / p} y\right)$. The quotient is a 3 -manifold called the lens space $L_{p, q}$.

A 3-manifold $M$ is called Seifert fibered if there is a $C^{\infty}$ morphism $f: M \rightarrow F$ to a topological surface such that every fiber is a circle. It turns out that $M$ is an $S^{1}$-bundle except over finitely many points where the local structure is given by one of the normal forms

$$
f_{c, d}: S^{1} \times D^{2} \rightarrow D^{2} \quad \text { where } \quad f_{c, d}(u, z)=u^{c} z^{d}
$$

$S^{1} \subset \mathbb{C}$ is the unit circle with coordinate $u, D^{2} \subset \mathbb{C}$ the closed unit disc with coordinate $z$ and $c, d$ are relatively prime integers satisfying $0<c<d$.

1.3. Moduli of Real Varieties. There has also been lot of work in describing all topological types represented by real algebraic varieties of a specific type. The case of plane curves is one of the Hilbert problems and quite a lot is known about them.

In the surface case, the first significant result was the classification of real cubic surfaces by [Schläfli1863]. [Zeuthen1874, Klein1876] studied plane curves of degree 4 and their bitangents, which is essentially equivalent to degree 2 Del Pezzo surfaces (though they related them to cubic surfaces only). The first systematic study was done in [Comessatti13] and [Comessatti14]. These papers presented a breakthrough whose extent has not been appreciated for over 50 years. The monograph [Segre42] treats cubic surfaces in detail.

The prototype of classification results that one would like is the following. In some form it has been known to [Comessatti14].

THEOREM 18. Let $F_{1}, F_{2}$ be smooth, projective, real algebraic surfaces which are rational over $\mathbb{R}$. Then $F_{1}$ can be deformed to $F_{2}$ through a family of smooth, projective, real algebraic surfaces iff

1. $F_{1}(\mathbb{C})$ and $F_{2}(\mathbb{C})$ are homeomorphic, and 
2. $F_{1}(\mathbb{R})$ and $F_{2}(\mathbb{R})$ are homeomorphic.

The case of surfaces which are rational over $\mathbb{C}$ is quite a bit harder. First of all, there is an easy exception given by the two surfaces

$$
F_{1}=Q^{3,0} \times \mathbb{P}^{1}, \quad F_{2}=Q^{3,0} \times Q^{3,0}
$$

where $Q^{3,0}$ is the empty conic $\left(x^{2}+y^{2}+z^{2}=0\right) \subset \mathbb{P}^{2}$. Thus we have to work with the more precise topological information given by the action of complex conjugation on the set of complex points. It turns out that the analog of (18) holds in all cases.

TheOREM 19. [Degtyarev-Kharlamov01] The deformation types of real algebraic surfaces which are rational over $\mathbb{C}$ are described by topological data, namely, by the action of complex conjugation on the set of complex points.

Similar results have been obtained for other classes of surfaces:

THEOREM 20. The deformation types of real algebraic surfaces are described by topological data in the following cases:

1. (Abelian surfaces) In this case the deformation type is determined by $F(\mathbb{R})$ alone. This is equivalent to the easy classification of real curves of genus 2.

2. (Hyperelliptic surfaces) [Catanese-Frediani00] In this case the deformation type is determined by the (algebraic) fundamental group.

3. (K3 surfaces) [Nikulin79, Kharlamov78] In this case the deformation type is determined by the action of complex conjugation on $H^{2}(F(\mathbb{C}), \mathbb{Z})$.

4. (Enriques surfaces) [DIK00] In this case the deformation type is determined by the actions of complex conjugation and of the fundamental group on $H^{2}(F(\mathbb{C}), \mathbb{Z})$ where $F$ is the K3-cover of the Enriques surface.

The proof of (20.4) is especially long and we end up with over 200 cases.

While these results are very nice, one should not expect that the topology determines the deformation type in many cases. This fails already for plane curves. Nonetheless, one can hope that similar results remain true for special classes of varieties in higher dimensions as well. Aside from the easy case of Abelian varieties, nothing is known.

NotATION 21. A real algebraic variety is an algebraic variety defined by real equations. Sometimes I write $X_{\mathbb{R}}$ instead of $X$ to emphasize that we are over $\mathbb{R}$. This is consistent with the usage in current algebraic geometry books (for instance [Hartshorne77, Shafarevich72]). This is, however, different from the definition of [BCR87] which essentially considers the germ of a variety along its real points.

If $X$ is a variety over $\mathbb{R}$ then $X(\mathbb{R})$ denotes its set of real points with the Euclidean topology and $X(\mathbb{C})$ denotes its set of complex points as a complex manifold.

I try to systematically distinguish the latter from $X_{\mathbb{C}}:=X \times_{\operatorname{Spec} \mathbb{R}} \operatorname{Spec} \mathbb{C}$, which is the complexification of $X$. One should also keep in mind the distinction between $X_{\mathbb{R}}$ and $X(\mathbb{R})$. The first is a scheme over $\mathbb{R}$, thus among its closed points we find all conjugate pairs of complex points of $X_{\mathbb{C}}$, but $X(\mathbb{R})$ is the collection of the real points only.

$\mathbb{P}^{n}$ stands for projective $n$-space (as a variety over $\mathbb{Q}$ ). Thus $\mathbb{P}_{\mathbb{R}}^{n}$ is projective $n$-space as a scheme over $\mathbb{R}$ and $\mathbb{P}^{n}(\mathbb{R})$ is the set of real points, customarily denoted by $\mathbb{R P P}^{n}$.

When talking about a point, curve or vector bundle etc. on a real variety $X$, these are, by definiton, objects defined over $\mathbb{R}$. I will always explicitly mention if we are considering a point, curve or vector bundle etc. on $X_{\mathbb{C}}$. 
If $Z \subset X_{\mathbb{C}}$ is a subscheme, then $\bar{Z} \subset X_{\mathbb{C}}$ denotes the subscheme defined by conjugate equations. Then $Z+\bar{Z} \subset X_{\mathbb{C}}$ can be defined by real equations and it can be viewed as a subscheme of $X_{\mathbb{R}}$. That is, there is a unique subscheme $Z_{\mathbb{R}} \subset X_{\mathbb{R}}$ such that $Z_{\mathbb{R}} \times_{\operatorname{Spec} \mathbb{R}} \operatorname{Spec} \mathbb{C}=Z+\bar{Z}$.

A property of $X$ (irreducible, normal, smooth etc.) always refers to the scheme theoretic property of $X$. Thus if $X$ is smooth then it is smooth not just at all points of $X(\mathbb{R})$, but also at all complex points. When talking about irreducibility (normality etc.) of $X_{\mathbb{C}}$, I say $X$ is "geometrically irreducible" or "irreducible over $\mathbb{C}$ ". In some cases this does not matter (for instance $X$ is normal or smooth iff $X_{\mathbb{C}}$ is) but in other cases the two versions are different ( $X$ may be irreducible but not geometrically irreducible).

The logical continuation of this terminology is to say that two irreducible $\mathbb{R}$ schemes $X, Y$ are birational iff there is a map $f: X \rightarrow Y$ defined over $\mathbb{R}$ which is birational. Similarly, $X$ is rational iff it is birational to $\mathbb{P}_{\mathbb{R}}^{n}$ over $\mathbb{R}$. Many authors, however, use the word "rational" to mean "geometrically rational". To avoid confusion, I will sometimes use the expressions rational over $\mathbb{R}$ and rational over $\mathbb{C}$.

Let $X \subset \mathbb{P}^{n}$ be a variety over $\mathbb{C}$ and $\bar{X}$ the variety defined by conjugate equations. The disjoint union of $X$ and $\bar{X}$ is invariant under conjugation, and so there is a real variety $Y_{\mathbb{R}}$ such that $Y_{\mathbb{C}} \cong X \cup \bar{X}$. Such real varieties are not particularly interesting since the theory of $Y_{\mathbb{R}}$ over $\mathbb{R}$ is equivalent to the theory of $X$ over $\mathbb{C}$. Thus it is reasonable to restrict our attention to real varieties which are geometrically irreducible.

Of course, during a proof we may run into a subvariety of $Y_{\mathbb{R}}$ which is geometrically reducible, and these have to be dealt with appropriately. Thus we can not ignore such varieties completely.

DEFINITION 22 (Kodaira dimension).

Let $X$ be a smooth projective variety and let $K_{X}$ denote its canonical bundle. That is, $K_{X}$ is the highest exterior power of the cotangent bundle. The Kodaira dimension of $X$, denoted by $\kappa(X)$ measures the growth rate of the spaces of global sections $H^{0}\left(X, K_{X}^{\otimes m}\right)$. To be precise, we set

$$
\kappa(X)=-\infty \Leftrightarrow H^{0}\left(X, K_{X}^{\otimes m}\right)=0 \quad \forall m>0 .
$$

If $H^{0}\left(X, K_{X}^{\otimes m}\right) \neq 0$ for some $m>0$ then, as it turns out, there is a unique integer $0 \leq \kappa(X) \leq \operatorname{dim} X$ such that

$$
0<\limsup \frac{\operatorname{dim} H^{0}\left(X, K_{X}^{\otimes m}\right)}{m^{\kappa(X)}}<\infty .
$$

(In fact, one expects that for all nonzero values of $H^{0}\left(X, K_{X}^{\otimes m}\right)$ the above quotient converges to a rational number. This number should be a very basic measure of the variety but even its existence is unknown in dimensions $\geq 4$.)

It is also conjectured that $\kappa(X)=-\infty$ iff $X$ is uniruled (that is, there is a generically finite map $\mathbb{P}^{1} \times Y \rightarrow X$ where $\left.\operatorname{dim} Y=\operatorname{dim} X-1\right)$. This is known only in dimension $\leq 3$.

\section{Minimal Models of Real Algebraic Surfaces}

This section is a warm up to the proof of (11). We study the topology of real algebraic surfaces, using methods that are applicable in dimension 3 . The 
main result is the following strengthening of (10). (This is not to hard obtain from topological considerations but the present proof shows the way to the 3 -dimensional results in sections 3 and 4.)

THEOREM 23. Let $F$ be a smooth, projective surface over $\mathbb{R}$ such that $F_{\mathbb{C}}$ is rational. Then one of the following holds:

0. $F(\mathbb{R})=\emptyset$.

1. $F(\mathbb{R}) \sim S^{1} \times S^{1}$.

2. $F(\mathbb{R}) \sim \# r_{1} \mathbb{R}^{2} \uplus \cdots \uplus \# r_{m} \mathbb{R}^{2}$ for some $r_{1}, \ldots, r_{m} \geq 0$.

All these cases do occur. (Note that $S^{2}=\# 0 \mathbb{R} \mathbb{P}^{2}$ by convention.)

We start with the first steps of the general minimal model program.

Definition 24. Let $X$ be a variety over a field $k$. A 1 -cycle on $X$ is a formal linear combination $C=\sum c_{i} C_{i}$, where the $C_{i} \subset X$ are irreducible, reduced and proper curves. A 1 -cycle is called effective if $c_{i} \geq 0$ for every $i$.

Two 1-cycles $C, C^{\prime}$ are numerically equivalent if $(C \cdot D)=\left(C^{\prime} \cdot D\right)$ for every Cartier divisor $D$ on $X$. 1-cycles with real coefficients modulo numerical equivalence form a vectorspace, denoted by $N_{1}(X) . N_{1}(X)$ is finite dimensional by the Theorem of the base of Néron-Severi (cf. [Hartshorne77, p.447]). Its dimension, denoted by $\rho(X)$, is called the Picard number of $X$.

Effective 1-cycles generate a cone $N E(X) \subset N_{1}(X)$. Its closure in the Euclidean topology $\overline{N E}(X) \subset N_{1}(X)$ is called the cone of curves of $X$.

If $K_{X}$ is Cartier (or at least some multiple of $K_{X}$ is Cartier) then set

$$
\overline{N E}(X)_{K \geq 0}:=\left\{z \in \overline{N E}(X) \mid\left(z \cdot K_{X}\right) \geq 0\right\} .
$$

Let $V \subset \mathbb{R}^{n}$ be a closed convex cone. For $v \in V$, a ray $\mathbb{R}^{\geq 0} v \subset V$ is called extremal if $u, u^{\prime} \in V, u+u^{\prime} \in \mathbb{R}^{\geq 0} v$ implies that $u, u^{\prime} \in \mathbb{R}^{\geq 0} v$. Intuitively: $\mathbb{R}^{\geq 0} v$ is an edge of $V$.

An extremal ray $\mathbb{R}^{\geq 0} z \subset \overline{N E}(X)$ is called $K_{X}$-negative if $\left(z \cdot K_{X}\right)<0$. This does not depend on the choice of $z$ in the ray.

We use the following description, due to [Mori82], of the cone of curves of smooth varieties over $\mathbb{C}$. See also [Kollár96, Kollár-Mori98] for proofs.

THEOREM 25 (Cone Theorem). Let $X$ be a smooth projective variety over an algebraically closed field. Then there are rational curves $C_{i} \subset X$ such that

$$
\overline{N E}(X)=\overline{N E}(X)_{K \geq 0}+\sum \mathbb{R}^{\geq 0}\left[C_{i}\right],
$$

and the $\mathbb{R}^{\geq 0}\left[C_{i}\right]$ are $K_{X}$-negative extremal rays of $\overline{N E}(X)$.

Let now $X$ be a smooth projective variety over $\mathbb{R}$. If $C$ is a 1 -cycle on $X_{\mathbb{C}}$ then $C+\bar{C}$ is a 1 -cycle on $X$, and every 1 -cycle on $X$ arises this way, at least with rational or real coefficients. Thus (25) immediately gives:

Theorem 26 (Cone Theorem over $\mathbb{R}$ ). Let $X$ be a smooth projective variety over $\mathbb{R}$. Then there are rational curves $C_{i} \subset X_{\mathbb{C}}$ such that

$$
\overline{N E}(X)=\overline{N E}(X)_{K \geq 0}+\sum \mathbb{R}^{\geq 0}\left[C_{i}+\bar{C}_{i}\right],
$$

and the $\mathbb{R}^{\geq 0}\left[C_{i}+\bar{C}_{i}\right]$ are $K_{X}$-negative extremal rays of $\overline{N E}(X)$. 
Proof. There is one point that we need to be careful about. Namely, it happens frequently that $C_{i}$ gives an extremal ray but $C_{i}+\bar{C}_{i}$ does not. So we have to throw away some of the $C_{i}$ appearing in (25).

THEOREM 27 (Contraction theorem). Let $X$ be a smooth projective variety over $\mathbb{R}$ and $R=\mathbb{R}^{\geq 0}\left[C_{i}+\bar{C}_{i}\right]$ a $K_{X}$-negative extremal ray of $\overline{N E}(X)$. Then there is a unique morphism, called the contraction of $R, \operatorname{cont}_{R}: X \rightarrow Y$ such that

1. $Y$ is normal and cont $_{R}$ has connected fibers.

2. An irreducible curve $D \subset X$ is mapped to a point in $Y$ iff $[D] \in R$.

The above theorems are sufficient to complete the minimal model program for surfaces. The key point is the classification of all contractions. This is stated after a definition.

Definition 28. Let $S$ be a smooth projective surface over a field $k . S$ is called a Del Pezzo surface if $S$ is geometrically irreducible and $-K_{S}$ is ample. It is called minimal (over $k$ ) if $\rho(S)=1$.

$S$, together with a morphism to a smooth curve $f: S \rightarrow B$ is called a conic bundle if every fiber is isomorphic to a plane conic. A conic bundle is called minimal if $\rho(S)=2$.

The geometric description and meaning of the extremal rays occurring in (26) is given in the next result:

THEOREM 29. Let $F$ be a smooth projective geometrically irreducible surface over $\mathbb{R}$ and $R=\mathbb{R}^{\geq 0}\left[C_{i}+\bar{C}_{i}\right] \subset \overline{N E}(F)$ a $K_{F}$-negative extremal ray. Then $R$ can be contracted $f: F \rightarrow F^{\prime}$, and we obtain one of the following cases:

(B) (Birational) $F^{\prime}$ is a smooth projective surface over $\mathbb{R}$ and $\rho\left(F^{\prime}\right)=\rho(F)-1$. $F$ is the blow up of $F^{\prime}$ at a closed point $P$. We have two cases:

(a) $P \in F^{\prime}(\mathbb{R})$, or

(b) $P$ is a pair of conjugate points.

(C) (Conic bundle) $B:=F^{\prime}$ is a smooth curve, $\rho(F)=2$ and $F \rightarrow B$ is a conic bundle. The fibers $f^{-1}(P): P \in B(\mathbb{C})$ are smooth, except for an even number of ponts $P_{1}, \ldots, P_{2 m} \in B(\mathbb{R}) .\left(K_{F}^{2}\right)=8(1-g(B))-2 m$.

(D) (Del Pezzo surface) $F^{\prime}$ is a point, $\rho(F)=1,-K_{F}$ is ample.

Proof. The adjunction formula says that $2 g(C)-2=\left(C^{2}\right)+\left(C \cdot K_{F}\right)$. In our case $\left(C \cdot K_{F}\right)<0$, so $-2<\left(C^{2}\right)$. We start with the low values of $\left(C^{2}\right)$.

If $\left(C^{2}\right)=-1$ and $C=\bar{C}$ then the contraction of $C$ in $F_{\mathbb{C}}$ is defined over $\mathbb{R}$, thus $F$ is the blow up of a surface at a real point. (This is Castelnuovo's contraction theorem, cf. [Hartshorne77, V.5.7].)

If $\left(C^{2}\right)=-1, C$ and $\bar{C}$ are disjoint, then we can contract them simultaneously over $\mathbb{R}$ to obtain $f: F \rightarrow F^{\prime}$ which is an isomorphism near $F(\mathbb{R})$.

If $\left(C^{2}\right)=-1$ and $(C \cdot \bar{C})=1$ or $\left(C^{2}\right)=(C \cdot \bar{C})=0$, then $C+\bar{C}$ has selfintersection 0 . It is relatively easy to see that $C+\bar{C}$ is a fiber of a conic bundle.

Over $\mathbb{C}$ we can contract one of the components of every singular fiber to obtain a minimal ruled surface. The selfintersection number of the canonical class of a minimal ruled surface is $8(1-g(B))$, and each singular fiber drops this number by 1 . We see in (33) that the number of singular fibers is even.

We are left with the cases when the selfintersection of $(C+\bar{C})$ is positive. By an a easy lemma (cf. [Kollár-Mori98, 1.21]), this implies that $\overline{N E}(F)$ is 1dimensional, hence $-K_{F}$ is ample. 
As a consequence we obtain the minimal model program (MMP for short) for real algebraic surfaces:

THEOREM 30 (MMP for real surfaces). Let $F$ be a smooth projective geometrically irreducible surface over $\mathbb{R}$. Then there is a sequence of morphisms

$$
F=F_{0} \stackrel{f_{0}}{\rightarrow} F_{1} \rightarrow \cdots F_{m-1} \stackrel{f_{m}-1}{\rightarrow} F_{m}=F^{*}
$$

such that each $f_{i}: F_{i} \rightarrow F_{i+1}$ is a birational contraction as in (29.B) and $F^{*}$ satisfies precisely one of the following properties:

(M) (Minimal model) $K_{F^{*}}$ is nef. (That is, it has nonnegative intersection number with every curve in $F^{*}$.)

(C) (Conic bundle) $F^{*}$ is a conic bundle over a curve $f: F^{*} \rightarrow B$. In particular, $\rho\left(F^{*}\right)=2$

(D) (Del Pezzo surface) $\rho\left(F^{*}\right)=1$ and $-K_{F^{*}}$ is ample.

Proof. We do the steps of (29.B) as long as we can. $\rho\left(F_{i+1}\right)=\rho\left(F_{i}\right)-1$, so eventually we reach $F^{*}=F_{m}$ where we can not perform a contraction as in (29.B). If $K_{F^{*}}$ is nef then we have a minimal model.

If $K_{F^{*}}$ is not nef, then by (29) we can perform a contraction as in (29.C-D). This gives our last two cases.

REMARK 31 . In the above proofs we had to establish several times that certain line bundles on $F_{\mathbb{C}}$ are defined over $\mathbb{R}$. This is frequently a quite subtle point.

Let $X$ be a scheme over $\mathbb{R}$ and $L$ a line bundle on $X$. Then $L_{\mathbb{C}}$ is a line bundle on $X_{\mathbb{C}}$ and $L_{\mathbb{C}} \cong \bar{L}_{\mathbb{C}}$. Thus if $M$ is a line bundle on $X_{\mathbb{C}}$ and $M \neq \bar{M}$, then $M$ is not the complexification of a real line bundle.

On the other hand, if $M \cong \bar{M}$ then $M$ is the complexification of a real line bundle, provided $X(\mathbb{R}) \neq \emptyset$. The latter condition is necessary. (For instance, consider the curve $C \cong\left(x^{2}+y^{2}+z^{2}\right) \subset \mathbb{P}^{2}$ over $\mathbb{R}$ and let $M$ be a degree 1 line bundle on $C_{\mathbb{C}}$. Then $M \cong \bar{M}$ but $M$ is not the complexification of a real line bundle.)

Let $X$ be a scheme over $\mathbb{R}$ and $M$ a line bundle on $X_{\mathbb{C}}$ such that $M \cong \bar{M}$. Then $M^{\otimes 2}$ is always the complexification of a real line bundle.

In is quite straightforward to follow the MMP for real surfaces from the topological point of view. The main results are already in

[Comessatti14] (though many people found some of his proofs very hard to follow).

Notation 32. $M \uplus N$ denotes the disjoint union of $M$ and $N$. $\uplus r N$ denotes the disjoint union of $r$ copies of $N . M \# N$ denotes the connected sum of two manifolds $M$ and $N$ (which are assumed to have the same dimension). \#rN denotes the connected sum of $r$ copies of $N$. (By definition, \#0M= $S^{\operatorname{dim} M}$.) $M \sim N$ denotes that $M$ and $N$ are homeomorphic.

One can give a complete topological description of the various contractions in (29):

THEOREM 33. Let $F$ be a smooth projective geometrically irreducible surface over $\mathbb{R}$ and $R \subset \overline{N E}(F)$ a $K_{F}$-negative extremal ray. The following is the topological description of the corresponding contraction:

(B) (Birational) $F$ is the blow up of $F^{\prime}$ at a closed point $P$. We have two cases: 
(a) If $P \in F^{\prime}(\mathbb{R})$ then $F(\mathbb{R}) \sim F^{\prime}(\mathbb{R}) \# \mathbb{R} \mathbb{P}^{2}$.

(b) If $P$ is a pair of conjugate points then $F(\mathbb{R}) \sim F^{\prime}(\mathbb{R})$.

(C) (Conic bundle) $f: F \rightarrow B$ is a conic bundle with singular fibers $f^{-1}\left(P_{1}\right), \ldots, f^{-1}\left(P_{2 m}\right)$. Then

$$
F(\mathbb{R}) \sim \uplus m S^{2} \uplus N_{1} \uplus \cdots \uplus N_{b},
$$

where $b$ is less than or equal to the number of connected components of $B(\mathbb{R})$ which do not contain any of the points $P_{i}$ and each $N_{i}$ is either a torus or a Klein bottle.

(D) (Del Pezzo surface) There are 4 cases:

(a) If $\left(K_{F}^{2}\right)=9$ then $F(\mathbb{R}) \sim \mathbb{R P P}^{2}$.

(b) If $\left(K_{F}^{2}\right)=8$ then $F(\mathbb{R}) \sim S^{2}$.

(c) If $\left(K_{F}^{2}\right)=2$ then $F(\mathbb{R}) \sim \uplus 4 S^{2}$.

(d) If $\left(K_{F}^{2}\right)=1$ then $F(\mathbb{R}) \sim \mathbb{R P P}^{2} \uplus 4 S^{2}$.

Proof. Blowing up replaces a point with all tangent directions through that point. So we remove a disc and put in an interval bundle over $S^{1}$ whose boundary is connected. This is a Möbius strip and so $F(\mathbb{R}) \sim F^{\prime}(\mathbb{R}) \# \mathbb{R} \mathbb{P}^{2}$.

In the conic bundle case, let $M \sim S^{1}$ be a connected component of $B(\mathbb{R})$. If none of the $P_{i}$ lie on $M$ then $F(\mathbb{R}) \rightarrow B(\mathbb{R})$ is an $S^{1}$-bundle over $M$, this gives either a torus, a Klein bottle or the empty set. If $k$ of the points $P_{1}, \ldots, P_{k} \in M \sim S^{1}$ correspond to singular fibers then, after reindexing, they divide $M$ into $k$ intervals $\left[P_{i}, P_{i+1}\right]$ (subscript $\left.\bmod k\right) . F(\mathbb{R})$ is alternatingly empty or a copy of $S^{2}$ over the intervals. Thus $k$ is even.

In the Del Pezzo case, let us prove first that the value of $\left(K_{F}^{2}\right)$ is one of those listed.

These cases appear in the proof of (30) in 3 different ways:

1. $\left(C^{2}\right)=-1$ and $r:=(C \cdot \bar{C}) \geq 2$,

2. $\left(C^{2}\right)=0$ and $r:=(C \cdot \bar{C}) \geq 1$, or

3. $\left(C^{2}\right)>0$.

In the first case

$$
\left((C+\bar{C})^{2}\right)=-2+2(C \cdot \bar{C})=2 r-2>0 .
$$

By an a easy lemma (cf. [Kollár-Mori98, 1.21]), this implies that $\overline{N E}(F)$ is 1dimensional, hence $-K_{F} \equiv a(C+\bar{C})$ for some $a>0$. $\left(-K_{F} \cdot(C+\bar{C})\right)=2$, thus

$$
(C+\bar{C}) \equiv(1-r) K_{F} \quad \text { and } \quad 2 r-2=(1-r)^{2}\left(K_{F}^{2}\right)
$$

This gives the possibilities $r=2,\left(K_{F}^{2}\right)=2$ or $r=3,\left(K_{F}^{2}\right)=1$.

If $\left(C^{2}\right)=0$ and $r:=(C \cdot \bar{C}) \geq 1$ then a computation as above gives that $8=r\left(K_{F}^{2}\right)$, which allows too many cases. It is better to consider this geometrically.

By (25), $C$ is a fiber of a $\mathbb{P}^{1}$-bundle $g: F_{\mathbb{C}} \rightarrow D$ over $\mathbb{C}$ and $\bar{C}$ is a (possibly multiple) section of $g$. Thus $D$ is rational. By the classification of minimal ruled surfaces, either $F_{\mathbb{C}} \cong \mathbb{P}^{1} \times \mathbb{P}^{1}$ and we are done by (34), or $g$ has a unique section $E$ with negative selfintersection. $E$ is then defined over $\mathbb{R}$, thus $\rho(F)=2$, a contradiction.

We are left with the case when $\left(C^{2}\right)>0$. This is actually mostly a complex question and the theory gives us that $F_{\mathbb{C}} \cong \mathbb{C P}^{2}$. Let $L$ be a line in $F_{\mathbb{C}} \cong \mathbb{C P}^{2}$. Then $\bar{L}$ is another line and $L$ and $\bar{L}$ intersect in a unique point, which is therefore 
real. We can get another real point, and so also a real line. Thus $\mathcal{O}_{F}(1)$ is defined over $\mathbb{R}$ and $F \cong \mathbb{R P}^{2}$.

We are also done with (33.D.a-b). The cases $\left(K_{F}^{2}\right)=2,1$ are considerably harder. They follow from (92) and (95).

In the next lemma, set

$$
Q^{p, q}:=\left(x_{1}^{2}+\cdots+x_{p}^{2}=x_{p+1}^{2}+\cdots+x_{p+q}^{2}\right) \subset \mathbb{P}^{p+q-1} .
$$

LEMMA 34. Let $F$ be a smooth projective surface over $\mathbb{R}$ such that $F_{\mathbb{C}} \cong \mathbb{P}^{1} \times \mathbb{P}^{1}$. Then one of the following holds:

1. $F \cong Q^{2,2} \cong Q^{2,1} \times Q^{2,1}, \rho(F)=2$ and $F(\mathbb{R}) \sim S^{1} \times S^{1}$,

2. $F \cong Q^{3,1}, \rho(F)=1$ and $F(\mathbb{R}) \sim S^{2}$,

3. $F \cong Q^{4,0} \cong Q^{3,0} \times Q^{3,0}, \rho(F)=2$ and $F(\mathbb{R})=\emptyset$,

4. $F \cong Q^{3,0} \times \mathbb{P}^{1}, \rho(F)=2$ and $F(\mathbb{R})=\emptyset$.

Proof. Let $C \subset F_{\mathbb{C}}$ be one of the rulings. Then $\bar{C}$ is another ruling, thus either $(C \cdot \bar{C})=0$ or $(C \cdot \bar{C})=1$.

If $(C \cdot \bar{C})=0$ then the linear system $|C+\bar{C}|$ is defined over $\mathbb{R}$ and maps $F$ onto a conic. Similarly for the other rulings, thus $F$ is the product of two conics. All 3 possibilities are listed.

If $(C \cdot \bar{C})=1$ then $\mathcal{O}_{F}(C+\bar{C})$ is a line bundle on $F$ which is of type $\mathcal{O}_{F_{\mathbb{C}}}(1,1)$ over $\mathbb{C}$. Thus its global sections embed $F$ as a quadric. $Q^{3,1}$ is the only quadric not yet accounted for.

Using (33) it is easy to determine which 2-manifolds occur as $F(\mathbb{R})$ for geometrically rational surfaces $F$.

35 (Proof of (23)). Apply the MMP over $\mathbb{R}$ to get $F=F_{1} \rightarrow F_{2} \rightarrow \cdots$. We prove the theorem by induction on the number of blow ups in the sequence. If $F_{i} \rightarrow$ $F_{i+1}$ is the inverse of the blowing up of a real point, then $F_{i}(\mathbb{R}) \sim F_{i+1}(\mathbb{R}) \# \mathbb{R}^{2}$. If $F_{i} \rightarrow F_{i+1}$ is the inverse of the blowing up of a pair of conjugate points, then $F_{i}(\mathbb{R}) \sim F_{i+1}(\mathbb{R})$. The induction works since $\left(S^{1} \times S^{1}\right) \# \mathbb{R}^{2} \sim \# 3 \mathbb{R}^{2}$.

Thus we are reduced to one of the following two cases:

1. $F$ has a conic bundle structure $F \rightarrow B$, or

2. $F$ is Del Pezzo and $\rho(F)=1$.

In the first case, $B_{\mathbb{C}} \cong \mathbb{C P}^{1}$ since $F_{\mathbb{C}}$ is rational. Thus either $B(\mathbb{R})=\emptyset$ and so $F(\mathbb{R})=\emptyset$, or $B \cong \mathbb{R}^{P^{1}}$. Thus $F(\mathbb{R})$ is the torus, the Klein bottle or the empty set if there are no singular fibers and $F(\mathbb{R}) \sim \uplus m S^{2}$ if there are $2 m>0$ singular fibers by (33.C).

In the second case we use (33.D).

REMARK 36. The hardest part of the proof of (33) is the still unfinished business of identifying the topology of Del Pezzo surfaces with $\left(K^{2}\right)=1,2$. It is very easy to establish that these cases give only finitely many topological types. Thus if we want to know the above theorems up to finitely many possible exceptions, then we are already done.

A similar situation accounts for the finitely many exceptions in (11). In 3dimensions I do not see how to settle the missing cases. 


\section{The Minimal Model Program for Real 3-folds}

In the surface case, the contraction of an extremal ray always results in a smooth variety. This is unfortunately not the case in higher dimensions. One of the main conceptual developments in higher dimensional birational geometry is the realization that we have to live with singularities. The smallest class that we need is called terminal singularities. A precise definition is given later (57), here let us accept that this class exists and go on stating the minimal model program.

Very roughly, the contractions of extremal rays can be classified into 3 types.

Definition 37. Let $g: X \rightarrow Y$ be an extremal contraction.

We say that $g$ is of fiber type if $\operatorname{dim} Y<\operatorname{dim} X$.

We say that $g$ is a divisorial contraction if the exceptional set $\operatorname{Ex}(g)$ is the support of Cartier divisor. In this case $\operatorname{Ex}(g)$ is irreducible over $K$.

We say that $g$ is a small contraction if $\operatorname{dim} \operatorname{Ex}(g) \leq \operatorname{dim} X-2$.

REMARK 38. A fiber type contraction is an end of a minimal model program. In effect we reduced the study of $X$ to the study of $Y$ and the fibers of $g$. Notethat $\operatorname{dim} Y=0$ is allowed, in which case we may not have achieved much. However, in this case $-K_{X}$ is ample, thus we need to develop a theory of these rather special class of varieties.

A divisorial contraction produces a new variety $Y$ of the same dimension. It turns out that the singularities are usually not worse than for $X$ and we can continue the program with $Y$. The Picard number drops by 1 , so this step can not be repeated forever.

A small contraction produces a very badly singular variety $Y$, and something radically new needs to be done. This operation, called a flip, is defined next.

Definition 39. Let $f: X \rightarrow Y$ be a small $K_{X^{-}}$-negative extremal contraction. A variety $X^{+}$together with a proper birational morphism $f^{+}: X^{+} \rightarrow Y$ is called a flip of $f$ if

1. $K_{X+}$ is $\mathbb{Q}$-Cartier,

2. $K_{X+}$ is $f^{+}$-ample, and

3. the exceptional set $\operatorname{Ex}\left(f^{+}\right)$has codimension at least two in $X^{+}$.

By a slight abuse of terminology, the rational map $\phi: X \rightarrow X^{+}$is also called a flip. A flip gives the following diagram:

$$
\begin{array}{rll}
X & \stackrel{\phi}{\rightarrow} & X^{+} \\
-K_{X} \text { is } f \text {-ample } & \searrow \swarrow & K_{X+} \text { is } f^{+} \text {-ample } \\
& &
\end{array}
$$

It is not hard to see that a flip is unique and the main question is its existence.

We are ready to state the 3-dimensional MMP over an arbitrary field:

THEOREM 40 (MMP over $K$ ). Let $X$ be a smooth projective 3-fold defined over a field $K$ (of characteristic zero). Then there is a sequence

$$
X=X_{0} \stackrel{f_{0}}{\rightarrow} X_{1} \rightarrow \cdots \rightarrow X_{i} \stackrel{f_{i}}{\rightarrow} X_{i+1} \rightarrow \cdots \stackrel{f_{n-1}}{\rightarrow} X_{n}=: X^{*}
$$

with the following properties

1. Each $X_{i}$ is a projective 3-fold over $K$ with terminal singularities (57). 
2. Each $f_{i}$ is either a $K_{X}$-negative divisorial extremal contraction or the flip of a $K_{X}$-negative small extremal contraction.

3. One of the following holds for $X^{*}$ :

(a) $K_{X^{*}}$ is nef (that is $\left(C \cdot K_{X^{*}}\right) \geq 0$ for any curve $\left.C \subset X^{*}\right)$, or

(b) there is a fiber type extremal contraction $X^{*} \rightarrow Z$.

The most important result about the finer workings of the MMP we need is the classification of terminal 3-fold singularities over nonclosed fields, established in [Kollár98b], building on the earlier complex classification. First let us fix our notation.

Notation 41 . For a field $K=\mathbb{R}$ or $K=\mathbb{C}$ let $K\left\{x_{1}, \ldots, x_{n}\right\}$ denote the ring of those formal power series which converge in some neighborhood of the origin.

For a power series $F, F_{d}$ denotes the degree $d$ homogeneous part. The multiplicity, denoted by mult $_{0} F$, is the smallest $d$ such that $F_{d} \neq 0$. If we write a power series as $F_{\geq d}$ then it is assumed that its multiplicity is at least $d$.

For $F \in \mathbb{R}\left\{x_{1}, \ldots, x_{n}\right\}$ let $(F=0)$ denote the germ of its zero set in $\mathbb{C}^{n}$ with its natural real structure. I always think of it as a complex analytic germ with a real structure and not just as a real analytic germ in $\mathbb{R}^{n}$.

$(F=0) / \frac{1}{n}(a, b, c, d)$ means the quotient of the hypersurface singularity $(F=0)$ by the group action $(x, y, z, t) \mapsto\left(\epsilon^{a} x, \epsilon^{b} y, \epsilon^{c} z, \epsilon^{d} t\right)$ where $\epsilon$ is a primitive $n$th root of unity. In all of our cases the quotient has a natural real structure.

THEOREM 42. [Kollár98b] Let $X$ be a real algebraic or analytic 3-fold and $0 \in$ $X(\mathbb{R})$ a real point. Then $X$ has a terminal singularity (57) at 0 iff a neighborhood of $0 \in X$ is real analytically equivalent to one of the following:

$$
\begin{array}{ll}
\text { name } & \quad \text { equation } \\
c A_{0} & (t=0) \\
c A_{1} & \left(x^{2}+y^{2} \pm z^{2} \pm t^{m}=0\right) \\
c A_{\geq 1}^{+} & \left(x^{2}+y^{2}+g_{\geq 3}(z, t)=0\right) \\
c A_{>1}^{-} & \left(x^{2}-y^{2}+g_{\geq 3}(z, t)=0\right) \\
c D_{4} & \left(x^{2}+f_{\geq 3}(y, z, t)=0\right), \text { where } f_{3} \neq l_{1}^{2} l_{2} \text { for linear forms } l_{i} \\
c D_{>4} & \left(x^{2}+y^{2} z+f_{\geq 4}(y, z, t)=0\right), \\
c E_{6} & \left(x^{2}+y^{3}+y g_{\geq 3}(z, t)+h_{\geq 4}(z, t)=0\right), \text { where } h_{4} \neq 0 \\
c E_{7} & \left(x^{2}+y^{3}+y g_{\geq 3}(z, t)+h_{\geq 5}(z, t)=0\right), \text { where } g_{3} \neq 0 \\
c E_{8} & \left(x^{2}+y^{3}+y g_{\geq 4}(z, t)+h_{\geq 5}(z, t)=0\right), \text { where } h_{5} \neq 0 \\
c A_{0} / n & (t=0) / \frac{1}{n}(r,-r, 1,0) \text { where } n \geq 2 \text { and }(n, r)=1 \\
c A_{1} / 2 & \left(x^{2}+y^{2} \pm z^{n} \pm t^{m}=0\right) / \frac{1}{2}(1,1,1,0) \text { where } \min \{n, m\}=2 \\
c A_{\geq 1}^{+} / 2 & \left(x^{2}+y^{2}+f_{\geq 3}(z, t)=0\right) / \frac{1}{2}(1,1,1,0) \\
c A_{>1}^{-} / 2 & \left(x^{2}-y^{2}+f_{\geq 3}(z, t)=0\right) / \frac{1}{2}(1,1,1,0) \\
c A / n & (x y+f(z, t)=0) / \frac{1}{n}(r,-r, 1,0) \text { where } n \geq 3 \text { and }(n, r)=1 \\
c A x / 2 & \left(x^{2} \pm y^{2}+f_{\geq 4}(z, t)=0\right) / \frac{1}{2}(0,1,1,1) \\
c A x / 4 & \left(x^{2} \pm y^{2}+f_{\geq 2}(z, t)=0\right) / \frac{1}{4}(1,3,1,2) \\
c D / 2 & \left(x^{2}+f_{\geq 3}(y, z, t)=0\right) / \frac{1}{2}(1,1,0,1) \\
c D / 3 & \left(x^{2}+f_{\geq 3}(y, z, t)=0\right) / \frac{1}{3}(0,1,1,2) \text { where } f_{3}(0,0,1) \neq 0 \\
c E / 2 & \left(x^{2}+y^{3}+f_{\geq 4}(y, z, t)=0\right) / \frac{1}{2}(1,0,1,1)
\end{array}
$$

In order to understand the birational steps of the real MMP, let us first look at some examples of contractions in 3-manifold theory.

43 (The topology of contractions of 3-manifolds). 
Let $M$ be a compact 3-manifold and $S \subset M$ a closed 2-dimensional subcomplex. Let $\pi: M \rightarrow N=N(M, S)$ denote the map that collapses $S$ to a point and is a homeomorphism outside $S$. Thus $N$ may have a single nonmanifold point at $P:=f(S)$. We would like to know how to reconstruct $M$ from knowing $N$ and $S$.

(43.1) For every 3-manifold $M$ there is a 2-dimensional subcomplex $S \subset M$ such that $N(M, S)$ is a bouquet of spheres. We can even choose $S$ to be a union of spheres intersecting transversally.

(43.2) For every 3-manifold $M$ and every $g \gg 1$ there is a surface $S \subset M$ of genus $g$ such that $N(M, S)$ is a union of two copies of $T_{g}$ where $T_{g}$ is the connected sum of $g$ solid tori with the boundary collapsed. Thus the precise knowledge of $N$ and of $S$ tells us nothing about $M$.

(43.3) Assume that $S$ has only finitely many nonmanifold points and $N(M, S)$ is the bouquet of 3-manifolds. Then a rather straightforward topological argument (cf. [Kollár99a, 5.7]) shows that $M$ can be obtained from $N$ by repeated application of the following operations:

1. separating the components of $N$,

2. taking connected sums of connected components,

3. taking connected sum with $S^{1} \times S^{2}$,

4. taking connected sum with $S^{1} \tilde{\times} S^{2}$,

5. taking connected sum with $\mathbb{R}^{3}$.

If we think of $M \rightarrow N$ as the real part of a morphism of 3-folds with exceptional set $E$ then $S=E(\mathbb{R})$ has only finitely many nonmanifold points if $E$ is a normal surface. Unfortunately, there does not seem to be any method to factor any birational morphism into steps which contract only one normal surface at a time. Thus we end up deriving very precise descriptions of the steps of the MMP and reading off normality of the exceptional surface at the end. In some cases normality fails, but then we have good enough control of the situation to see that everyhting is nice topologically.

From the technical point of view the key point is to control the singularities that occur during a minimal model program.

44 (Which singularities are complicated?).

The singularities can cause trouble in two ways.

First, a singularity can be complicated algebraically. It is by now well understood that the most intricate features of the 3-dimensional MMP occur only in the presence of quotient singularities. Thus if we can exclude these, the MMP is much more manageable. Hence we should exclude the last 10 types of singularitites on the list (42).

Second, a singularity can be complicated topologically. An isolated singularity on a real algebraic threefold is locally a cone over a surface, called the link of the singularity. As long as this surface is the union of spheres, we are dealing essentially with a manifold and the usual methods of manifold theory work well. The situation becomes quite complicated if the link is a high genus surface. In this case the real variety is very far from a manifold and it becomes nearly impossible to follow the minimal model program topologically. 
Singularities which are very similar algebraically can have very different topological behaviour. For instance, the link of

$$
x^{2}+y^{2}+\prod_{i=1}^{n}\left(z-a_{i} t\right)=0
$$

is a union of $n$ spheres and the link of

$$
x^{2}-y^{2}+\prod_{i=1}^{n}\left(z-a_{i} t\right)=0
$$

is a surface of genus $n$. These two singularities are complex analytically equivalent.

Assume that we start with a smooth real algebraic threefold $X$ and we run the minimal model program. Let $X_{i}$ be one of the intermediate steps. One can think of $X$ as a resolution of singularities of $X_{i}$. This is not completely correct since the flips in the program are not everywhere defined, thus $X \rightarrow X_{i}$ is not always a morphism. This however effects only finitely many curves and for our current purposes it does not matter much. Thus, at least philosophycally we are asking the following:

QUESTION 45. Which of the 3-dimensional, real, terminal singularities admit a resolution with orientable real part.

REMARK 46. One can ask similar questions about different topological properties. For instance, the proof of (13) requires the study of those real, terminal singularities that admit a resolution whose real part satisfies the conditions (13.2.a-c).

One can also ask for stronger conditions. For instance it is of interest to study those resolutions where the real part has vanishing homology groups.

In order to understand (45) let us study the analogous surface question first.

47 (Resolutions of surface singularities).

The topological study of resolutions of surface singularities is made quite easy by the fact that every surface singularity has a unique minimal resolution. That is, every other resolution is obtained from the minimal one by blowing up points (which is connected sum by $\mathbb{R P}^{2}$ at the topological level). Thus a surface singularity has an orientable resolution iff the minimal resolution is orientable. Thus we have a concrete model to check.

48 (Resolutions of threefold singularities).

The topological study of resolutions of threefold singularities is more complicated since we do not have a unique minimal resolution. To be precise, starting with a threefold $X$, there is a nearly unique partial resolution $X^{t} \rightarrow X$ where $X^{t}$ has terminal singularities. The resolutions of $X^{t}$ are, however, far from being unique. This does not help us at all, since we start with a threefold with terminal singularities and the first step $X^{\iota} \rightarrow X$ is the identity. Thus we need a way to compare two resolutions. One way is the following.

Definition 49. Let $X$ be a variety, $f: Y \rightarrow X$ a proper birational morphism and $E \subset Y$ an exceptional divisor. Let $g: Z \rightarrow X$ be another proper birational morphism. The composite $h:=g^{-1} \circ f: Y \rightarrow Z$ is birational and the image $h(E)$ is either a subset of codimension at least 2 , or it is a subset of codimension 1 and $E \rightarrow h(E)$ is a birational map. In the latter case we say that $E$ appears on $Z$ and that $E$ and $h(E)$ are equivalent exceptional divisors. 
Assume now that everything is defined over $\mathbb{R}$ and that $E(\mathbb{R})$ represents a nontrivial homology class in $Y(\mathbb{R})$. Then there is a loop $L=\operatorname{im}\left[S^{1} \rightarrow Y(\mathbb{R})\right]$ which produces a nonzero intersection number with $E(\mathbb{R})$. The loop can be moved away from the codimension 2 set where $h$ is not defined, thus $h(E(\mathbb{R}))$ and $h(L)$ have nonzero intersection number again. (We have to be a little more careful if $h$ collapses some divisors to curves or points.) Thus we may be able to carry information about the homology of one resolution to the homology of another resolution.

This is of course useless if every exceptional divisor on $Y$ is collapsed by $h$. Thus we are led to the following:

QUESTION 50. Which divisors appear on every resolution?

Let us illustrate this first with the case of smooth points. Here the simplest resolution is the identity, thus we should ask about all nontrivial birational maps $f: Y \rightarrow X$.

EXAMPLE 51 (Blow ups of smooth varieties). If $\operatorname{dim} X=2$ and $f^{-1}$ is not defined at a point $P \in X$ then $f$ factors through the blow up $B_{P} X$. Thus we have a unique nontrivial minimal blow up.

If $\operatorname{dim} X=3$ then we have many more choices. For instance, we can blow up a point $P$ or we can blow up any smooth curve through $P$. These already give infinitely many different choices but they correspond to just 2 different types.

Thus we can still hope to get a complete list of proper birational morphisms $g_{\lambda}: Z_{\lambda} \rightarrow X$ such that every proper birational morphism $f: Y \rightarrow X$ factors through at least one of them as

$$
f: Y \stackrel{h}{\rightarrow} Z_{\lambda} \stackrel{g_{\lambda}}{\rightarrow} X
$$

As a first attempt, we should restrict ourself to the smooth case. As we saw, the list of $Z_{\lambda} \rightarrow X$ should include the blow ups of points and smooth curves.

There are, however, further possibilities. For instance, choose local coordinates $(x, y, z)$ at $P$ and blow up a curve $P \in C \subset X$ with local equations

$$
\left(z=x^{2} \pm y^{2}+(\text { higher degree terms })=0\right) \text {. }
$$

We get $X_{1} \rightarrow X$ where $X_{1}$ has a unique singular point. Next we blow up the singular point. We get $X_{2} \rightarrow X_{1}$ with exceptional divisor $F \subset X_{2}$. It turns out that $X_{2}$ is smooth. Let $E \subset B_{P} X$ be the exceptional divisor of the ordinary blow up. By explicit computation we see that $E$ and $F$ are equivalent. $E \cong \mathbb{P}^{2}$ and $F$ is a quadric, so they are not isomorphic. It is easy to see that $X_{2}$ does not dominate any of the blow ups considered first.

There are many more such examples, and we do not even have a conjecture about what a complete list $Z_{\lambda} \rightarrow X$ should look like. It is quite probable that the list is enormous, nearly as big as the list of all birational morphisms $Z \rightarrow X$. Thus the list is unlikely to be useful.

There are two related ideas that seem to work better.

52 (Last steps of the MMP).

Given a variety $X$, we may ask for a list of all divisorial contractions $Z_{\lambda} \rightarrow X$. This would give some information on all resolutions of $X$ since the MMP starting with any resolution would go through one of the $Z_{\lambda} \rightarrow X$. This is a quite interesting question. Unfortunately, the answer is not known for most terminal singularities. 
A list like this should be useful in many other questions and finding it is one of the interesting open problems of 3-dimensional birational geometry.

The case when $X$ is smooth was solved very recently by [Kawakita00].

A second, less ambitious option is to ask for a list of exceptional divisors that appear on every resolution.

53 ("Minimal" exceptional divisors).

The right formulation of the question is the following. Given a variety $X$, find all exceptional divisors $E_{i}$ which appear on every resolution $f: Y \rightarrow X$ whose exceptional set is a divisor.

(Many of the singularities on the list (42) admit a resolution whose exceptional set is a curve. In the MMP, this problem is usually dealt with by requiring all singularities to be $\mathbb{Q}$-factorial. This is a technical condition which takes care of the problem completely. It has no effect on the general framework of the proof, so we ignore it for the rest of these notes.)

The following is a prototypical result in this direction.

ThEOREM 54. [Sun95] Let $f: Y \rightarrow X$ be a birational morphism between smooth threefolds.

1. If $f^{-1}$ is not defined along a curve $C \subset X$ then the exceptional divisor of $B_{C} X \rightarrow X$ appears on $Y$.

2. If $f^{-1}$ is not defined at finitely many points $P_{i} \in X$ then the exceptional divisor of $B_{P_{i}} X \rightarrow X$ appears on $Y$.

For toric varieties a complete description of these divisors is given in [Bouvier98].

Our aim is to develop a version of this result for the real terminal singularities occurring in (42). To do this, we have to have a way of measuring how soon exceptional divisors appear on blow ups.

55 (The hierarchy of exceptional divisors).

Let $X$ be any normal variety and assume that its canonical class $K_{X}$ is a Cartier divsior, or at least some multiple of $K_{X}$ is Cartier. This holds, for instance, for all hypersurface singularities or for all quotients of hypersurface singularities. These cover all the cases in (42).

Let $f: Y \rightarrow X$ be any proper birational map. Then we can write $f^{*} K_{X}$ as

$$
f^{*} K_{X}=K_{Y}+\sum_{i} a\left(E_{i}, X\right) E_{i}
$$

where the summation runs through all exceptional divisors. The (rational) number $a\left(E_{i}, X\right)$ is called the discrepancy of $E_{i}$ with respect to $X$. In practice we want to look at the discrepancies of exceptional divisors on all proper birational maps $Y \rightarrow X$. It is easy to see that equivalent divisors have the same discrepancy.

ExAmple 56. Let $f: Y \rightarrow X$ be a birational morphism between smooth varieties. Then $a(E, X)$ is the order of vanishing of the Jacobian of $f$ along $E$. Thus $a(E, X) \geq 1$ for every exceptional divisor. It is also not hard to see that $a(E, X)=1$ iff $f(E) \subset X$ has codimension 2 and $E$ is equivalent to the exceptional divisor of the blow up of $f(E)$.

DEFinition 57. We say that $X$ has terminal singularities if the discrepancy of every exceptional divisor is positive. 
In the terminal case, there is a useful sufficient condition which guarantees that an exceptional divisor appears on every resolution.

Proposition 58. Let $X$ be a variety with terminal singularities and $E$ an exceptional divisor

1. If $a(E, X)<1$ then $E$ appears on every resolution of $X$ (whose exceptional set is a divisor).

2. If $a(E, X)=1$ then

(a) either $E$ appears on every resolution of $X$ (whose exceptional set is a divisor), or

(b) $E$ is the exceptional divisor of the blow up of a codimension 2 subvariety $V \subset X$.

Proof. Let $g: Z \rightarrow X$ be any resolution of singularities. $h: g^{-1} \circ f: Y \rightarrow Z$ is a rational map. We are done if $E$ appears on $Z$. Otherwise, $E$ is an exceptional divisor of $h$ and we have

$$
\begin{aligned}
f^{*} K_{X} & =h^{*} K_{Z}+\sum_{i} a\left(E_{i}, Y\right) E_{i} \\
& =h^{*}\left(g^{*} K_{X}\right)+h^{*}\left(\sum_{j} a\left(F_{j}, X\right) F_{j}\right)+\sum_{i} a\left(E_{i}, Y\right) E_{i},
\end{aligned}
$$

where $E_{i}$ are the exceptional divisors of $h$ and $F_{j}$ the exceptional divisors of $g . E$ appears among the $E_{i}$ and its discrepancy is at least 1 by (56). $E$ also may appear in the effective divsior $h^{*}\left(\sum_{j} a\left(F_{j}, X\right) F_{j}\right)$, necessarily with positive coefficient. This already shows (1).

If $a(E, X)=1$ then $h(E)$ is not a subset of any exceptional divisor of $g$ and $E$ corresponds to the blow up of a codimension 2 subvariety $V \subset Y$ by (56). Thus $E$ also corresponds to the blow up of the codimension 2 subvariety $g(V) \subset X$. (Here we use that the excptional set has pure codimension 1.)

Now our task is relatively straightforward:

59 (Main computation).

For any of the singularities in (42), [Kollár99a] finds all exceptional divisors with discrepancy $\leq 1$ and computes if the presence of these divisors contradicts the orientability of the real part or not.

The algebraic part is closely related to the earlier works of [Markushevich96] and [Hayakawa99].

For most cases this is rather easy to do and one can exclude many singularities this way. A number of singularities are, however, not excluded by divisors of discrepancy $\leq 1$. Then one needs secondary computations of divisors with discrepancy $\leq 2$ and in one case one even needs to understand divisors with discrepancy $\leq 3$.

In these cases one has to take the real structure carefully into consideration. Also, the divisors with discrepancy $>1$ do not need to appear on every resolution, so additional considerations are needed.

At the end we get the following:

THEOREM 60. Let $X$ be a smooth, projective, real algebraic 3 -fold and assume that $X(\mathbb{R})$ satisfies the 3 conditions (13.2.a-c).

Let $X_{i}$ be any of the intermediate steps of the $M M P$ over $\mathbb{R}$ starting with $X$ and $0 \in X_{i}(\mathbb{R})$ a real point. Then a neighborhood of $0 \in X_{i}$ is real analytically equivalent to one of the following standard forms: 
1. (cA $\left.A_{0}\right)$ Smooth point.

2. $\left(c A_{>0}^{+}\right)\left(x^{2}+y^{2}+g_{\geq 2}(z, t)=0\right)$, where $g$ is not everywhere negative in a punctured neighborhood of 0 .

3. $\left(c E_{6}\right)\left(x^{2}+y^{3}+\left(z^{2}+t^{2}\right)^{2}+y g_{\geq 4}(z, t)+g_{\geq 6}(z, t)=0\right)$.

The above points of type $c E_{6}$ form a codimension 6 family in the space of all $c E_{6}$ singularities. They all do occur. Points of type $c A_{>0}^{+}$occur for many choices of $g$.

REMARK 61. It should be emphasized that our computations limit only the real singular points on the $X_{i}$. There are no restrictions on the complex singularities, except that they appear in conjugate pairs. Thus the full complexity of the $3-$ dimesional MMP appears but it does not influence the real part much.

It is also very convenient to have a quick way of determining the orientability of the real part using algebraic data.

62 (Degrees of line bundles over $\mathbb{R}$ and $\mathbb{C}$ ).

Let $B$ be a smooth projective curve over $\mathbb{C}$ and $L$ a line bundle on $B$. Let $s$ be a nonzero meromorphic section of $L$. The number of zeros minus the number of poles of $s$ on $B$ (counted with multiplicity) is called the degree of $L$. Let $Y$ be a smooth projective variety over $\mathbb{C}$ and $L$ a line bundle on $Y$. For any curve $B \subset Y$ the degree of $\left.L\right|_{B}$ is defined. It is also called the intersection number of $B$ and $L$ and denoted by $(B \cdot L)$.

Let $A \sim S^{1}$ be a compact 1-dimensional manifold and $L$ a real line bundle on $M$. Let $s$ be a nonzero section of $L$. The number of zeros of $s$ on $A$ (counted with multiplicity) makes sense only $\bmod 2$. If $M$ is a compact manifold and $L$ a real line bundle on $M$ then for any 1-cycle $A \subset M$ we obtain the $\mathbb{Z}_{2}$-valued intersection number of $A$ and $L$. It is denoted by $(A \cap L)$. (To be precise, I should write $\left(A \cap w_{1}(L)\right)$ where $w_{1}(L)$ stands for the first Stiefel-Whitney class of $L$. This is a class in $H^{1}\left(X(\mathbb{R}), \mathbb{Z}_{2}\right)$ analogous to the first Chern class of a complex line bundle, cf. [Milnor-Stasheff74, Sec.4].)

Let now $X$ be a smooth projective variety over $\mathbb{R}, C \subset X$ a curve and $L$ a line bundle on $X$. We obtain two numbers:

$$
(C(\mathbb{R}) \cap L(\mathbb{R})) \text { and }\left(C_{\mathbb{C}} \cdot L_{\mathbb{C}}\right)
$$

What is the relationship between them?

To answer this, take a real meromorphic section $s$ of $L$ which has only finitely many zeros and poles on $C$. When we count the real zeros and poles of $s$ on $C(\mathbb{R})$, we miss the complex zeros and poles of $s$ on $C_{\mathbb{C}}$. Since $s$ is real, the complex zeros and poles come in conjugate pairs. Thus we conclude that

$$
(C(\mathbb{R}) \cap L(\mathbb{R})) \equiv\left(C_{\mathbb{C}} \cdot L_{\mathbb{C}}\right) \bmod 2,
$$

which is best possible since the left hand side is defined only mod 2 anyhow.

63 (Orientability of $X(\mathbb{R})$ and the canonical class).

Let $M$ be a differentiable manifold, $0 \in M$ a point and $x_{1}, \ldots, x_{n}$ local coordinates. A local orientation of $M$ at 0 is a choice of an $n$-form $f(x) d x_{1} \wedge \cdots \wedge d x_{n}$ with $f(0) \neq 0$ up to multiplication by a positive function. An orientation of $M$ is a nowhere zero global $n$-form on $M$, up to multiplication by a positive function. $n$-forms are sections of the real line bundle $\operatorname{det} T_{M}^{*}$. If $S^{1} \sim A \subset M$ is a loop then 
one can choose a consistent orientation of $M$ along $A \Leftrightarrow \operatorname{det} T_{M}^{*}$ has a nowhere zero section along $A \Leftrightarrow\left(\operatorname{det} T_{M}^{*} \cap A\right)=0$.

If $X$ is a smooth variety over $\mathbb{R}$ then $n$-forms appear as sections of the canonical line bundle. This proves that

$$
\operatorname{det} T_{X(\mathbb{R})}^{*} \cong K_{X}(\mathbb{R})
$$

In many cases this gives a way to decide if $X(\mathbb{R})$ is orientable or not.

Corollary 64. Let $X$ be a smooth projective variety over $\mathbb{R}$. Assume that there is a curve $C \subset X$ such that $\left(C \cdot K_{X}\right)$ is odd. Then $X(\mathbb{R})$ is not orientable.

Proof. We have proved above that

$$
\left(C(\mathbb{R}) \cap \operatorname{det} T_{X(\mathbb{R})}^{*}\right) \equiv\left(C \cdot K_{X}\right) \equiv 1 \bmod 2 .
$$

$C(\mathbb{R})$ may have several components, but along one of them $\operatorname{det} T_{X(\mathbb{R})}^{*}$ has odd degree, so we can not choose a consistent orientation along that component.

If $\left(C \cdot K_{X}\right)$ is even, then it can happen that $X(\mathbb{R})$ is not orientable along an even number of components of $C(\mathbb{R})$. In some cases we are still able to conclude orientability of $X(\mathbb{R})$ using stronger assumptions:

LEMMA 65. Let $X$ be a smooth projective variety over $\mathbb{R}$. Assume that $K_{X} \cong$ $L^{\otimes 2}$ for a real line bundle $L$. Then $X(\mathbb{R})$ is orientable.

EXAMPLE $66 . \mathbb{R P}^{n}$ is orientable iff $n$ is odd. Let $X \subset \mathbb{P}^{n}$ be a smooth hypersurface of degree $d$. Then $X(\mathbb{R})$ is orientable if $n-d$ is odd. $X(\mathbb{R})$ is not orientable if $n$ and $d$ are both odd. If $n$ and $d$ are both even, then $X(\mathbb{R})$ may or may not be orientable.

Once the singularities of the intermediate stages of the MMP are limited, a rather similar computation comes up with a list of possible steps of the MMP.

THEOREM 67. Let $X$ be a smooth, projective, real algebraic 3-fold such that $X(\mathbb{R})$ satisfies the conditions (13.2. $a-c)$.

Let $f_{i}: X_{i} \rightarrow X_{i+1}$ be any of the intermediate steps of the MMP over $\mathbb{R}$ starting with $X$. Then the induced map $f_{i}: X_{i}(\mathbb{R}) \rightarrow X_{i+1}(\mathbb{R})$ is everywhere defined and the following is a complete list of possibilities for $f_{i}$ :

1. ( $\mathbb{R}$-trivial) $f_{i}$ is an isomorphism in a (Zariski) neighborhood of the set of real points.

2. ( $\mathbb{R}$-small) $f_{i}: X_{i}(\mathbb{R}) \rightarrow X_{i+1}(\mathbb{R})$ collapses a 1 -complex to points.

3. (smooth point blow up) $f_{i}$ is the inverse of the blow up of a smooth point $P \in X_{i+1}(\mathbb{R})$.

4. (singular point blow up) $f_{i}$ is the inverse of a (weighted) blow up of a singular point $P \in X_{i+1}(\mathbb{R})$. There are two cases:

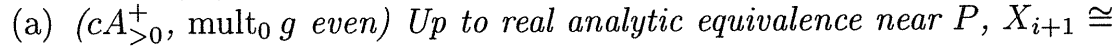
$\left(x^{2}+y^{2}+g_{\geq 2 m}(z, t)=0\right)$ where $g_{2 m}(z, t) \neq 0$ and $X_{i}$ is the weighted blow up $B_{(m, m, 1,1)} X_{i+1}$.

(b) ( $c A_{>0}^{+}$, mult $g$ odd) Up to real analytic equivalence near $P, X_{i+1} \cong$ $\left(x^{2}+y^{2}+g_{\geq 2 m+1}(z, t)=0\right)$ where $z^{2 m+1} \in g$ and $z^{i} t^{j} \notin g$ for $2 i+j<4 m+2$. $X_{i}$ is the weighted blow up $B_{(2 m+1,2 m+1,2,1)} X_{i+1}$. 
68 (End of the proof).

The previous consideration assure us that we understand the change of the topology of the real part through the birational steps of the MMP. If $\kappa(X)=-\infty$, then the last step is a fiber type contraction. Hence we still need to understand the topology of a variety $Y$ in the following 3 cases.

1. There is an extremal contraction $f: Y \rightarrow S$ to a surface. Here the general fiber is $\mathbb{P}^{1}$.

2. There is an extremal contraction $f: Y \rightarrow C$ to a curve. Here the general fiber is a Del Pezzo surface.

3. There is an extremal contraction $f: Y \rightarrow P$ to a point. Here $-K_{Y}$ is ample.

In the first case the general fiber of $Y(\mathbb{R}) \rightarrow S(\mathbb{R})$ is $S^{1}$ and it is reasonable to hope that $Y(\mathbb{R})$ is Seifert fibered. From the topological point of view this is quite delicate since the singular fibers can have a very substantial effect on the topology. Still, after careful computations in [Kollár99b] we obtain that $Y(\mathbb{R})$ is either Seifert fibered or a connected sum of lens spaces.

In the second case the general fiber is either a torus or a union of spheres. The tricky part is again to control the singular fibers. This was done in [Kollár00a].

Finally we are left with the case when $Y$ is a Fano variety, that is, $-K_{Y}$ is ample. Smooth Fano threefolds are completelly classified, but we can have conjugate pairs of complex quotient singularities. Fortunately, even these singular Fano threefolds form a bounded family by a theorem of [Kawamata92]. This is the main source of the finitely many exceptions.

A complete list of singular Fano threefolds is not known and there are no reasonable bounds about the number of families.

Even if the complex variety is well known, the topology of the real part may be rather hard to determine. Here is the simplest unsolved question:

Conjecture 69. Let $X \subset \mathbb{P}^{4}$ be a real hypersurface of degree 4. Then $X(\mathbb{R})$ $s$ not hyperbolic.

\section{The Nash Conjecture for Nonprojective Threefolds}

In this section we consider the Nash conjecture for nonprojectivite 3-folds. Allowing quasi projective varieties instead of projective ones does not help at all. Indeed, if $Y$ is quasi projective and $Y(\mathbb{R})$ is compact then $\bar{Y}(\mathbb{R})=Y(\mathbb{R})$ for every smooth compactification $\bar{Y} \supset Y$. To get something interesting, we should look at compact complex manifolds which can be obtained from $\mathbb{P}^{3}$ by a sequence of smooth blow ups and blow downs. This class of manifolds has been studied by many authors.

Definition 70. [Artin68, Moishezon67] A compact complex manifold $Y$ is called a Moishezon manifold or a (smooth) algebraic space if it is bimeromorphic to a projective variety.

A real Moishezon manifold or a real algebraic space is an algebraic space $Y$ with an antiholomorphic involution $\tau: Y \rightarrow Y$.

It is not hard to see that if $(Y, \tau)$ is a real algebraic space then there is a projective real algebraic variety $\left(Y^{\prime}, \tau^{\prime}\right)$ and a conjugation invariant bimeromorphic $\operatorname{map} \phi: Y \rightarrow Y^{\prime}$. 
By a result of Chow and Kodaira, every smooth Moishezon surface is projective (cf. [BPV84, IV.5]). The first smooth nonprojective examples in dimension 3 were found by Hironaka (see [Hartshorne77, App.B.3]).

Moishezon manifolds are quite close to projective varieties. In fact, as a general philosophy, if a property of projective varieties does not obviously involve projectivity then it should also be true for Moishezon manifolds. Thus it was quite a surpize to me that the Nash conjecture is true for Moishezon manifolds in a strong form.

Before explaining the method of the proof, we give a quick review of surgeries of 3-manifolds. As a general reference, see [Rolfsen76, Chap.2].

71 (Surgery on 3-manifolds).

Let $D^{2}$ be the unit disc $(|u| \leq 1) \subset \mathbb{C}_{u}$ and $S^{1}$ the unit circle $(|v|=1) \subset \mathbb{C}_{v}$. A three-manifold $T$ diffeomorphic to $D^{2} \times S^{1}$ is called a solid torus. The boundary of a solid torus

$$
\partial T \sim(|u|=1) \times(|v|=1) \sim S^{1} \times S^{1}
$$

is a torus. Any simple closed curve on the torus $S^{1} \times S^{1}$ is isotopic to one of the form

$$
C_{a, b}:=\operatorname{im}\left[(|z|=1) \rightarrow S^{1} \times S^{1}\right] \text { given by } z \mapsto\left(z^{a}, z^{b}\right) \text { for }(a, b)=1 .
$$

The meridian of a solid torus is any curve isotopic to $C_{ \pm 1,0}$. Note that these curves are generators of $\operatorname{ker}\left[\pi_{1}(\partial T) \rightarrow \pi_{1}(T)\right]$, so their isotopy class is well defined.

The correspondence

$$
\left(\begin{array}{cc}
a & b \\
c & d
\end{array}\right) \mapsto\left[(u, v) \mapsto\left(u^{a} v^{b}, u^{c} v^{d}\right)\right]
$$

gives an isomorphism

$$
G L(2, \mathbb{Z}) \cong \frac{\text { (diffeomorphisms of the torus) }}{\text { (modulo isotopy) }} .
$$

Up to isotopy, the diffeomorphisms of a solid torus are given by

$$
(u, z) \mapsto\left(u z^{m}, z^{ \pm 1}\right) \quad \text { or } \quad(u, z) \mapsto\left(\bar{u} z^{m}, z^{ \pm 1}\right), \quad \text { where } \quad m \in \mathbb{Z} .
$$

They correspond to the subgroup

$$
B(2, \mathbb{Z}):=\left(\begin{array}{cc} 
\pm 1 & * \\
0 & \pm 1
\end{array}\right) \subset G L(2, \mathbb{Z})
$$

Let $M$ be a 3-manifold and $L \subset M$ a knot. (That is, an embedded copy of $S^{1}$.) Assume that $M$ is orientable along $L . L$ has a tubular neighborhood which is a solid torus $T$ with bundary $\partial T \sim S^{1} \times S^{1}$. Set $N:=M \backslash \operatorname{Int} T$. We can think of $M$ as being glued together from these two pieces $M=T \cup N$. Let $\phi: \partial T \rightarrow \partial T$ be any orientation preserving diffeomorphism. We can glue together $T$ and $N$ using $\phi$ to obtain another 3-manifold $M_{\phi}:=T \cup_{\phi} N$. The operation that creates $M_{\phi}$ from $M$ is called a surgery along $L$. (If $\phi$ is orientation reversing, we can compose $\phi$ with an orientation reversing diffeomorphism of $T$ to see that we do not get anything new.) If we fix a diffeomorphism $h: T \sim D^{2} \times S^{1}$ then a diffeomorphism $\phi: \partial T \rightarrow \partial T$ corresponds to an element of $S L(2, \mathbb{Z})$.

Two diffeomorphisms of the torus that differ by a diffeomorphism of the solid torus give diffeomorphic surgeries. Computing representatives of the cosets $G L(2, \mathbb{Z}) / B(2, \mathbb{Z})$ we obtain the following: 
Proposition 72. The image of the meridian of $T$ under $\phi$ determines the surgery up to diffeomorphism.

We are especially interested in surgeries that can be realized algebraically. From the algebraic point of view the simplest surgery-type operations are flops. It turns out that we need only the simplest flop.

73 (Flops of $(-1,-1)$-curves).

Let $X$ be a complex 3 -manifold and $\mathbb{C P}^{1} \cong C \subset X$ a curve. Assume that the normal bundle of $C$ in $X$ is isomorphic to $\mathcal{O}_{C}(-1)+\mathcal{O}_{C}(-1)$. Let us blow up $C$ in $X$. We obtain $p_{1}: X_{1} \rightarrow X$ with exceptional divisor $Q \cong \mathbb{C P}^{1} \times \mathbb{C P}^{1}$. Moreover, the normal bundle $N_{Q, X_{1}}$ has intersection number -1 with both rulings of $Q$.

Thus $Q \subset X_{1}$ is symmetrical with respect to the two factors of $Q$ and by a result of [Nakano70] it can be contracted (if we allow $X^{\prime}$ to be an algebraic space) in the other direction to obtain $p_{2}: X_{1} \rightarrow X^{\prime}$. The bimeromorphic map $\phi:=p_{2} \circ p_{1}^{-1}: X \rightarrow X^{\prime}$ is called the flop of $C$. Frequently $X^{\prime}$ is also called a flop of $X$. Note that $X$ and $X^{\prime}$ have a completely symmetrical role in a flop.

Assume next that $X$ has a real structure and $C$ is also real and isomorphic to $\mathbb{P}^{1}$ over $\mathbb{R}$. Thus $C(\mathbb{R}) \sim S^{1}$. A tubular neighborhood of $S^{1} \sim C(\mathbb{R}) \subset X(\mathbb{R})$ is a trivial disc bundle over $S^{1}$, but the algebraic direct sum decomposition of the normal bundle gives that

$$
N_{C(\mathbb{R}) \mid X(\mathbb{R})} \cong \mathcal{O}_{C(\mathbb{R})}(-1)+\mathcal{O}_{C(\mathbb{R})}(-1)
$$

which is the sum of two Möbius bands.

We can identify $N_{C(\mathbb{R}) \mid X(\mathbb{R})}$ with a tubular neighborhood $T \supset C(\mathbb{R})$, then any of the direct summands $\mathcal{O}_{C(\mathbb{R})}(-1)$ corresponds to an embedded Möbius band $B \subset T$ with boundary $\partial B \subset \partial T$.

$X_{1}(\mathbb{R})$ is obtained from $X(\mathbb{R})$ by blowing up $C(\mathbb{R})$ which is contained in $B$. Thus the birational transform $\left(p_{1}\right)_{*}^{-1} B$ is isomorphic to $B$ and it intersects $Q(\mathbb{R})$ in a fiber of $p_{2}$. Hence the birational transform of $B$ in $X^{\prime}$ is a disc which intersects $C^{\prime}(\mathbb{R})$ transversally. Thus $X(\mathbb{R}) \mapsto X^{\prime}(\mathbb{R})$ is a surgery along $C(\mathbb{R})$ where $\partial B$ becomes the new meridian.

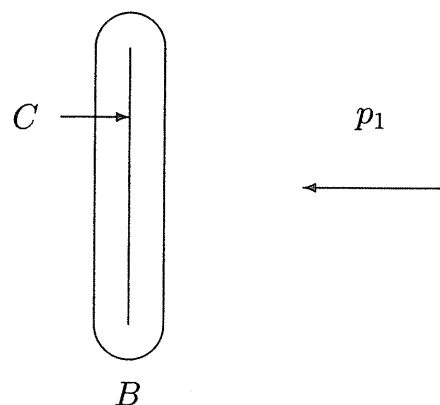

Möbius band

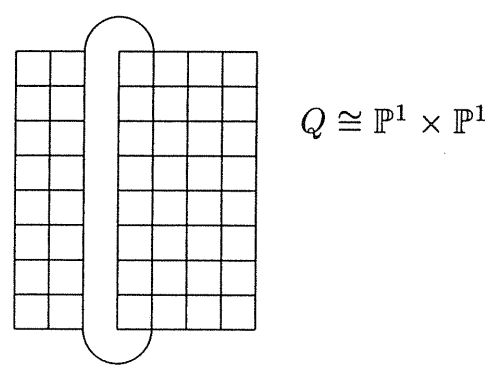

$p_{2}$

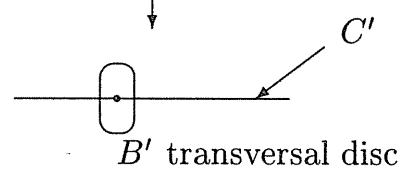


Definition 74. Let $M$ be a 3-manifold and $B \subset M$ an embedded Möbius band with center $L$. Assume that $M$ is orientable along $L$. By (72) $B$ defines a unique surgery $M \mapsto M^{\prime}$. We call $M^{\prime}$ the topological flop of $M$ along $B$.

The diffeomorphism class of $M^{\prime}$ is determined by the pair $(M, B)$ up to isotopy.

The relationship between algebraic and topological flops is summarized in the next result which is a direct consequence of our previous discussions.

Proposition 75. Let $X$ be a 3-dimensional, smooth, real, algebraic space and $\mathbb{P}^{1} \cong C \subset X$ a real curve. Assume that $N_{C \mid X} \cong \mathcal{O}_{C}(-1)+\mathcal{O}_{C}(-1)$. Let $B \subset X(\mathbb{R})$ be a Möbius band corresponding to one of the $\mathcal{O}_{C}(-1)$ summands and $X \rightarrow X^{\prime}$ the flop of $B$. Then $X^{\prime}(\mathbb{R})$ is diffeomorphic to the topological flop of $X(\mathbb{R})$ along $B$.

Now we can explain the main steps of the proof of (12).

76 (Idea of the proof of (12)).

1. First we find a normal form for 3-manifolds up to topological flops. This is a purely topological result which has been accomplished in [Benedetti-Marin92].

2. We realize each of the above normal forms by a 3-manifold $X(\mathbb{R})$ for some real projective algebraic variety $X$, birational to $\mathbb{P}^{3}$. In all but one case this was done in [Benedetti-Marin92]. In the last case this probably can not be done and we need a nonprojective example.

3. We realize every topological flop as a sequence of blow ups followed by an algebraic flop. Of course we need to choose the blow ups in such a way that they do not change the topology of the real points.

The first step of (76) in the orientable case relies on the following very nice result.

Theorem 77. [Benedetti-Marin92] Let $M_{1}, M_{2}$ be two compact orientable 3-manifolds. Then $M_{2}$ can be obtained from $M_{1}$ by a sequence of topological flops iff $h_{1}\left(M_{1}, \mathbb{Z}_{2}\right)=h_{1}\left(M_{2}, \mathbb{Z}_{2}\right)$.

Instead of explaining the proof, let me illustrate the method in a key step: how to untangle knots by flops.

Let $L \subset \mathbb{R}^{3}$ be a knot. It is known that each knot has a Seifert surface, that is, an embedded surface $S$ whose boundary is exactly $L$.

First we claim that we may assume $S$ to be nonorientable. Indeed, if we slide the handle on the left hand side of the picture to the edge of the Seifert surface (with 2 new holes) on the right, we get a new nonorientable Seifert surface of the knot $L$.
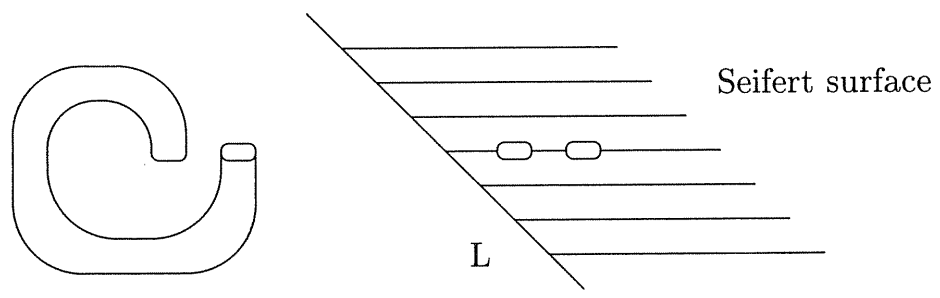
Every nonorientable surface with disk boundary is the connected sum of projective planes $\# k \mathbb{R P}^{2}$ with a disc removed. Let $C_{1}, \ldots, C_{k}$ be lines in the projective planes. The neighborhood of a line in $\mathbb{R P}^{2}$ is a Möbius band, so each $C_{i} \subset S$ corresponds to a topological flop of $\mathbb{R}^{3}$. Performing the flops contracts each $C_{i}$ to a point, thus the image of $S$ after all these flops is a disc. Hence $L$ becomes unknotted.

COROLlary 78. Let $X_{k}$ denote a rational variety obtained from the quadric $\left(x_{0}^{2}+x_{1}^{2}+x_{2}^{2}+x_{3}^{2}=x_{4}^{2}\right) \subset \mathbb{P}^{4}$ by blowing up $k$ real points. Then every compact orientable 3-manifold $M$ can be obtained from $X_{k}(\mathbb{R})$ (with $k=h_{1}\left(M, \mathbb{Z}_{2}\right)$ ) by a sequence of topological flops.

Proof. $X_{k}(\mathbb{R})$ is diffeomorphic to $S^{3} \# k \mathbb{R} \mathbb{P}^{3}$, hence it is orientable and $h_{1}\left(X_{k}(\mathbb{R}), \mathbb{Z}_{2}\right)=k$. We are done by $(77)$.

In the general case, we use the following consequence of the classification, which takes care of the first two steps in (76). The construction of the exceptional case is by a series of explicitly described blow ups and downs, see [Kollár00b, Sec.6] for details.

Theorem 79. [Benedetti-Marin92, Thm.B and Ex.C.5] Any compact 3manifold $M$ can be obtained by a sequence of topological flops from a 3-manifold $N$ such that either

1. $N$ can be obtained form $S^{3}$ by repeatedly blowing up points and simple closed curves, or

2. $N$ is a torus bundle over $S^{1}$ with monodromy $\left(\begin{array}{ll}0 & 1 \\ 1 & 1\end{array}\right) \in G L(2, \mathbb{Z})$.

A Möbius band $B \subset M$ with center $L$ can be thought of as a subline bundle $B^{\prime}$ of the normal bundle $N_{L \mid M}$. Thus, the algebraic version of a Möbius band should be a smooth, real, rational curve $\mathbb{P}^{1} \cong C \subset X$ and a real subline bundle (of odd degree) of the normal bundle $N_{C \mid X}$.

Taking into account the ultimate aim of producing algebraic flops, we would like to get a real subline bundle of degree -1 of the normal bundle $\mathcal{O}_{C}(-1) \hookrightarrow N_{C \mid X}$. The next theorem says that this is indeed possible.

THEOREM 80. Let $X$ be a compact, smooth, real, algebraic space which is birational to $\mathbb{P}^{3}$. Let $B \subset X(\mathbb{R})$ be an embedded Möbius band. Then there is

1. a smooth, real, rational curve $\mathbb{P}^{1} \cong C \subset X$ such that $C \cdot K_{X} \leq 0$, and

2. a real subline bundle $\mathcal{O}_{C}(-1) \cong B^{\prime} \subset N_{C \mid X}$ such that the corresponding Möbius band is isotopic to $B \subset X(\mathbb{R})$.

Idea of the proof. Any continuous function on $S^{1}$ can be approximated by trigonometric polynomials. Thus any map $S^{1} \rightarrow \mathbb{R}^{3}$ can be approximated by trigonometric polynomials, and we can even assume this to be a $C^{1}$-approximation. The trigonometric polynomials are exactly the polynomials $p(x, y)$ restricted to the circle. This way we see that any $S^{1} \subset \mathbb{R}^{3}$ can be approximated by the real points of a rational algebraic curve obtained as the image of the plane conic $x^{2}+y^{2}=1$. By carefully following the projective version, [Bochnak- $\mathbf{K}$ ucharz99] proves that with $X$ as above there are plenty of morphisms $f: \mathbb{P}^{1} \rightarrow X$ such that $f\left(\mathbb{R P}^{1}\right)$ is a very good approximation of $L$. A precise technical consequence of "plenty" is that $f^{*} T_{X}$ can be assumed to be as ample as we want. This implies that there will be 
many subline bundles $\mathcal{O}_{\mathbb{P}^{1}}(-1) \hookrightarrow N_{C \mid X}$, and we can realize $B \subset X(\mathbb{R})$ by the real points of a subline bundle $\mathcal{O}_{\mathbb{P}^{1}}(-1) \cong B^{\prime} \hookrightarrow N_{C \mid X}$.

A precise formulation of this is a bit tricky since there is no canonical choice for the trivialization of a tubular neighborhood in general. Thus some artificial choice has to be made and everything measured accordingly.

81 (Construction of algebraic flops).

Assume now that we have our real subline bundle $\mathcal{O}_{\mathbb{P}^{1}}(-1) \cong B^{\prime} \hookrightarrow N_{C \mid X}$. Let $Q^{\prime}$ be the quotient $Q^{\prime}:=N_{C \mid X} / B^{\prime} . \operatorname{deg} N_{C \mid X}=-K_{X} \cdot C-2 \geq-2$ by (80.1), thus $\operatorname{deg} Q^{\prime} \geq-1$. In order to flop, we need to achieve $\operatorname{deg} Q^{\prime}=-1$. This will be done by a blow up.

Let $D \subset X$ be a smooth, real, algebraic curve such that

1. $D \cap C$ is precisely $r$ points $P_{1}, \ldots, P_{r}$, and

2. the tanget vector of $D$ at every point $P_{i}$ lies in $B^{\prime} \subset N_{C \mid X}$.

Let us blow up $D$ to obtain $p: X_{1}:=B_{D} X \rightarrow X$ and set $C_{1}:=p_{*}^{-1} C$. There is an exact sequence

$$
0 \rightarrow B^{\prime} \rightarrow N_{C_{1} \mid X_{1}} \rightarrow Q^{\prime}\left(-\sum P_{i}\right) \rightarrow 0
$$

If $\operatorname{deg} Q^{\prime}\left(-\sum P_{i}\right)=\operatorname{deg} Q^{\prime}-r=-1$, the sequence splits and

$$
N_{C_{1} \mid X_{1}} \cong \mathcal{O}_{C_{1}}(-1)+\mathcal{O}_{C_{1}}(-1) \text {. }
$$

Thus $C_{1} \subset X_{1}$ can be flopped to get $X_{2}$. Topologically we want to flop a curve in $X(\mathbb{R})$, and this is achieved as long as $X_{1}(\mathbb{R})$ is diffeomorphic to $X(\mathbb{R})$. This can happen only if $D(\mathbb{R})=\emptyset$. There are plenty of real curves without real points, so this should be easy to achieve. The only additional problem is that in this case $D \cap C$ consists of conjugate point pairs, hence $r$ is even. Thus our construction works only if $\operatorname{deg} Q^{\prime}=-K_{X} \cdot C-1$ is odd.

$X(\mathbb{R})$ is orientable along $C(\mathbb{R})$, thus $w_{1}(X(\mathbb{R})) \cdot C(\mathbb{R})=0$ where $w_{1}$ denotes the first Stiefel-Whitney class (cf. [Milnor-Stasheff74]).

$$
c_{1}(X(\mathbb{C})) \cdot C \equiv w_{1}(X(\mathbb{R})) \cdot C(\mathbb{R}) \quad \bmod 2
$$

by $(62)$, hence $-K_{X} \cdot C=c_{1}(X(\mathbb{C})) \cdot C$ is even. Thus the only remaining question is the choice of $D$. This is actually trickier than it seems.

82 (General position curves in algebraic spaces).

If $X$ is a quasi projective variety, it is easy to construct a curve $D$ as above by taking the intersection of suitable hypersurfaces. On an algebraic space there may not exist any base point free linear system, so intersections of hypersurfaces are unlikely to produce the needed curve $D$. In fact, there are singular algebraic spaces with a unique singular point $P \in X$ such that every curve in $X$ passes through $P$. No such smooth examples are known and it is conjectured that this can not happen for smooth algebraic spaces. (See [Kollár91, 5.2.6] for the case when $\operatorname{dim} X \leq 3$.)

In our case we deal with Moishezon spaces birational to $\mathbb{P}^{3}$, and so they contain plenty of rational curves. The deformation spaces of rational curves are very well understood and standard techniques (see, for instance [Kollár-Mori98]) allow us to construct $D$.

\section{The Topology of Real Del Pezzo Surfaces}

In this section we describe the topological types of all real Del Pezzo surfaces. This is the last step needed to finish the proofs of (33) and of (23). 

maps.

We start with the description of some explicit isomorphisms and birational

DEFINITION 83. Let $F$ be a smooth real algebraic surface. A surface obtained from $F$ by blowing up $a$ real points and $b$ pairs of conjugate complex points (possibly infinitely near) is denoted by $(F, a, 2 b)$.

LEMMA 84. We have the following elementary birational equivalences between the minimal models in (29).

1. $\left(\mathbb{P}^{2}, 2,0\right) \cong\left(Q^{2,2}, 1,0\right)$.

2. $\left(\mathbb{P}^{2}, 0,2\right) \cong\left(Q^{3,1}, 1,0\right)$.

3. $\left(Q^{4,0}, 0,2\right)$ is isomorphic to the blow up of $Q^{3,0} \times \mathbb{P}^{1}$ at a pair of conjugate points on the same section $Q^{3,0} \times P, P \in \mathbb{R}^{1}$.

4. Any minimal conic bundle over a rational curve with 2 singular fibers is isomorphic to $\left(Q^{3,1}, 0,2\right)$.

Proof. In the first two cases we blow up the 2 points in $\mathbb{P}^{2}$ and then contract the line through them to get a quadric.

$Q^{4,0} \cong Q^{3,0} \times Q^{3,0}$, let $\pi_{1}$ be the first projection. The pencil of planes through the 2 points gives a map $p: Q^{4,0} \rightarrow \mathbb{P}^{1}$.

$$
\left(\pi_{1}, p\right): Q^{4,0} \rightarrow Q^{3,0} \times \mathbb{P}^{1} \quad \text { is birational }
$$

and becomes a morphism after blowing up the 2 points.

Finally assume that $F \rightarrow B$ is a minimal conic bundle over a rational curve with 2 singular fibers. By $(29 . C), B(\mathbb{R}) \neq \emptyset$, thus $B \cong \mathbb{P}^{1} . F_{\mathbb{C}}$ is the blow up of a minimal ruled surface $F^{\prime \prime}$ at 2 points. We can even assume that $F^{\prime \prime}$ has a section $E$ with negative selfintersection $\left(E^{2}\right)=-k$ and the two points are not on $E$. If $k \geq 2$ then all other sections of $F^{\prime \prime}$ have selfintersection at least 2, so $E \subset F$ is the unique section with negative selfintersection. Thus $E$ is defined over $\mathbb{R}$ and $F \rightarrow B$ is not minimal.

Thus $k=1$ and there is a unique section $E^{\prime} \subset F^{\prime \prime}$ such that $\left(E^{2}\right)=1$ and $E^{\prime}$ passes through the two blown up points. Let $\bar{E} \subset F$ be the birational transform. Then $E$ and $\bar{E}$ have to be conjugate. Contracting them gives the quadric $Q^{3,1}$.

Proposition 85. Let $F$ be a smooth real Del Pezzo surface with $F(\mathbb{R})=\emptyset$ whose minimal model has degree 8 or 9. Set $d:=\left(K_{F}^{2}\right)$. Then $d \in\{8,6,4,2\}$.

If $d=8$ then $F$ is isomorphic to either $Q^{3,0} \times Q^{3,0}$ or to $Q^{3,0} \times \mathbb{P}^{1}$.

If $d<8$ then $F$ is isomorphic to $Q^{3,0} \times \mathbb{P}^{1}$ blown up in $\frac{1}{2}(8-d)$ pairs of conjugate points.

Proof. Apply the MMP over $\mathbb{R}$ to obtain $F \rightarrow \cdots \rightarrow F^{*}$. If $F$ is Del Pezzo then so is $F^{*}$, and $\left(K_{F^{*}}^{2}\right) \geq\left(K_{F}^{2}\right)$. Hence in our case $F^{*}$ is either $Q^{3,0} \times Q^{3,0}$ or $Q^{3,0} \times \mathbb{P}^{1}$. By (84.3) any blow up of $Q^{3,0} \times Q^{3,0}$ at a pair of conjugate points is also a blow up of $Q^{3,0} \times \mathbb{P}^{1}$.

Proposition 86. Let $F$ be a smooth real Del Pezzo with $F(\mathbb{R}) \neq \emptyset$ whose minimal model has degree 8 or 9 . Set $d:=\left(K_{F}^{2}\right)$. Then $9 \geq d \geq 1$ and we have one of the following cases:

If $d=9$ then $F$ is isomorphic to $\mathbb{P}^{2}$.

If $d=8$ then $F$ is isomorphic to either $Q^{3,1}$ or to $Q^{2,2}$ or to $\mathbb{P}^{2}$ blown up at a real point.

If $d<8$ then $F$ is isomorphic to one of the following: 
1. $\mathbb{P}^{2}$ blown up at $a \geq 0$ real points and $b \geq 0$ pairs of conjugate points for some $a+2 b=9-\bar{d}$. Thus $F(\mathbb{R}) \sim \#(a+1) \mathbb{R P}^{2}$.

2. $Q^{3,1}$ blown up at $b=\frac{1}{2}(8-d)$ pairs of conjugate points (so $d$ is even). Thus $F(\mathbb{R}) \sim S^{2}$.

3. $Q^{2,2}$ blown up at $b=\frac{1}{2}(8-d)$ pairs of conjugate points (so $d$ is even). Thus $F(\mathbb{R}) \sim S^{1} \times S^{1}$.

Proof. The minimal model of such a surface is either $\mathbb{P}^{2}, Q^{3,1}$ or $Q^{2,2}$. By (84.1-2) any blow up of $Q^{3,1}$ or to $Q^{2,2}$ at a real point is also a blow up of $\mathbb{P}^{2}$.

The two propositions above account for all Del Pezzo surfaces of degrees $d \geq 5$. The results are summarized in the next statement:

COROLlaRY 87. The following table lists all topological types of the real points of Del Pezzo surfaces of degrees $9 \geq d \geq 5$.

\begin{tabular}{|c|l|}
\hline degree & \multicolumn{1}{|c|}{ topological types } \\
\hline 9 & $\mathbb{R P}^{2}$ \\
\hline 8 & $S^{2}$ or $S^{1} \times S^{1}$ or $\mathbb{R} \mathbb{P}^{2} \# \mathbb{R P}^{2}$ or $\emptyset$ \\
\hline 7 & $\mathbb{R P}^{2}$ or $\# 3 \mathbb{R P}^{2}$ \\
\hline 6 & $S^{2}$ or $S^{1} \times S^{1}$ or $\mathbb{R P} \mathbb{P}^{2} \# \mathbb{R P}^{2}$ or $\# 4 \mathbb{R} \mathbb{P}^{2}$ or $\emptyset$ \\
\hline 5 & $\mathbb{R P}^{2}$ or $\# 3 \mathbb{R P}^{2}$ or $\# 5 \mathbb{R} \mathbb{P}^{2}$ \\
\hline
\end{tabular}

The following result shows that odd degree Del Pezzo surfaces over $\mathbb{R}$ are relatively easy to understand:

Lemma 88. Every degree $2 d-1$ Del Pezzo surface $F$ over $\mathbb{R}$ with $\rho(F) \geq 2$ is the blow up of a degree $2 d$ Del Pezzo surface at a real point.

Proof. Since $\left(K_{F}^{2}\right)$ is odd, $F$ is not a minimal conic bundle. Thus $F$ is either the blow up of a degree $2 d$ Del Pezzo surface at a real point or the blow up of a degree $2 d+1$ Del Pezzo $F^{\prime}$ surface at a conjugate pair of complex points $P+\bar{P}$.

If $F^{\prime} \cong \mathbb{P}^{2}$ then let $L \subset \mathbb{P}^{2}$ be the line through the two points. Its birational transform on $F$ is a line.

Otherwise, $F^{\prime}$ is again not minimal by (29), hence $F^{\prime}$ contains a line $L$ over $\mathbb{R}$ by induction. $P, \bar{P} \notin L$ since otherwise the birational transform of $L$ on $F$ would have a nonnegative intersection number with $K_{F}$. Thus $L$ gives a real line on $F$.

Contracting a real line on $F$ we get a degree $2 d$ Del Pezzo surface.

This shows that the study of degree 3 Del Pezzo surfaces is reduced to the study of degree 4 cases. The classification of these two classes is summarized next. These results were obtained by [Schläfli1863], who actually worked directly with cubic surfaces.

COROLlARY 89. The following table lists all topological types of the real points of Del Pezzo surfaces of degrees 4 and 3.

\begin{tabular}{|c|l|}
\hline degree & topological types \\
\hline 4 & $S^{2}$ or $S^{1} \times S^{1}$ or $\mathbb{R} \mathbb{P}^{2} \# \mathbb{R P}^{2}$ or $\# 4 \mathbb{R P}^{2}$ or $\emptyset$ or $S^{2} \uplus S^{2}$ \\
\hline 3 & $\mathbb{R} \mathbb{P}^{2}$ or $\# 3 \mathbb{R}^{2}$ or $\# 5 \mathbb{R P}^{2}$ or $\# 7 \mathbb{R} \mathbb{P}^{2}$ or $S^{2} \uplus \mathbb{R} \mathbb{P}^{2}$ \\
\hline
\end{tabular}

Proof. As we noted above, it is sufficient to describe all degree 4 Del Pezzo surfaces. 
If a degree 4 Del Pezzo surface $F$ is obtained from a higher degree surface by blowing up then we are reduced to (87). Otherwise $F$ is a conic bundle over $\mathbb{P}^{1}$ with 4 singular fibers, thus $F(\mathbb{R}) \sim S^{2} \uplus S^{2}$.

Next we move to the harder cases of Del Pezzo surfaces of degrees 2 and 1.

Notation 90. Let $D \subset \mathbb{P}^{2}$ be a degree 4 smooth real curve. $D(\mathbb{R})$ divides $\mathbb{R}^{2}$ into connected open sets and precisely one of these is nonorientable (denoted by $\left.U_{D}\right)$. We choose an equation $f(x, y, z) \in \mathbb{R}[x, y, z]$ of $D$ such that $f$ is negative on $U_{D}$

We can associate two different degree 2 Del Pezzo surfaces to $D$. One is $F_{D}^{+}:=\left(u^{2}=f(x, y, z) \subset \mathbb{P}^{3}(1,1,1,2)\right.$ and the other $F_{D}^{-}:=\left(u^{2}=-f(x, y, z) \subset\right.$ $\mathbb{P}^{3}(1,1,1,2)$.

The correspondence $F_{D}^{+} \leftrightarrow F_{D}^{-}$is a natural involution on the space of degree 2 real Del Pezzo surfaces.

$D$ has 28 bitangents over $\mathbb{C}$ and over each bitangent of $D$ we get a pair of lines on $F_{D}^{ \pm}$. This gives a total of 56 lines.

The topological classification of degree 4 plane curves over $\mathbb{R}$ is very old, it is already contained in [Plücker1839]. (See [Viro90] for a recent survey of the study of low degree real plane curves.) This implies the topological classification of degree 2 real Del Pezzo surfaces. The following proposition summarizes these results.

Proposition 91. There are 6 topological types of degree 4 smooth real plane curves. Correspondingly there are 12 topological types of degree 2 real Del Pezzo surfaces. The following table gives the complete list. The types in the same row correspond to each other under $D \leftrightarrow F_{D}^{+} \leftrightarrow F_{D}^{-}$.

\begin{tabular}{|c|c|c|}
\hline$D(\mathbb{R})$ & $F_{D}^{+}(\mathbb{R})$ & $F_{D}^{-}(\mathbb{R})$ \\
\hline$\bigcirc \bigcirc \bigcirc$ & $\uplus 4 S^{2}$ & $\# 8 \mathbb{R}^{2}$ \\
\hline$\bigcirc \bigcirc \bigcirc$ & $\uplus 3 S^{2}$ & $\# 6 \mathbb{R P}^{2}$ \\
\hline$\bigcirc \bigcirc$ & $S^{2} \uplus S^{2}$ & $\# 4 \mathbb{R P}^{2}$ \\
\hline$\bigcirc$ & $S^{2}$ & $\# 2 \mathbb{R P}^{2}$ \\
\hline$\emptyset$ & $\emptyset$ & $\mathbb{R P}^{2} \uplus \mathbb{R P}^{2}$ \\
\hline$\bigcirc$ & $S^{1} \times S^{1}$ & $S^{2} \uplus \# 2 \mathbb{R P}^{2}$ \\
\hline
\end{tabular}

[Zeuthen1874] studied the bitangents of degree 4 plane curves. He proved the equivalence of (92.1) and (92.5). He understood the relationship between degree 4 plane curves and cubic surfaces. (Projecting a cubic surface from one of its points, the branch curve is a plane quartic. Equivalently, blowing up the cubic at a point we get a degree 2 Del Pezzo surface.) This is, however, not the natural thing to do from the modern viewpont. Most of (92) is proved in [Comessatti13].

THEOREM 92. Let $D \subset \mathbb{P}^{2}$ be a degree 4 smooth real curve. The following are equivalent:

1. All 28 bitangents of $D$ are real.

2. All 56 lines of $F_{D}^{-}$are real.

3. $F_{D}^{-}$is isomorphic to $\mathbb{P}^{2}$ blown up in 7 real points.

4. $F_{D}^{-}(\mathbb{R}) \sim \# 8 \mathbb{R}^{2}$.

5. $D(\mathbb{R}) \sim \uplus 4 S^{1}$.

6. $F_{D}^{+}(\mathbb{R}) \sim \uplus 4 S^{2}$. 


\section{7. $F_{D}^{+}$has Picard number 1 over $\mathbb{R}$.}

Proof. $(1) \Rightarrow(2)$ : A neighborhood of a line in $\mathbb{R P}^{2}$ is not orientable, thus any bitangent is contained in $U_{D}$ (except for the points of tangency). $f$ is negative on any bitangent and so $u^{2}=-f$ has real solutions, giving 56 real lines on $F_{D}^{-}$.

$(2) \Rightarrow(3)$ : Over $\mathbb{C}, F_{D}^{-}$is the blow up of $\mathbb{P}^{2}$ at 7 points, hence it has 7 disjoint lines. If all lines are real, we have 7 disjont real lines. Contracting these we get a Del Pezzo surface of degree 9 over $\mathbb{R}$. By (29.D) it is $\mathbb{P}_{\mathbb{R}}^{2}$.

$(3) \Rightarrow(4)$ : Topologically, each blowing up is connected sum with $\mathbb{R}^{2}$.

$(4) \Rightarrow(5)$ : This follows from (91).

$(5) \Rightarrow(6)$ : This also follows from (91).

$(6) \Rightarrow(7)$ : Assume to the contrary that $F_{D}^{+}$has Picard number $\geq 2$ over $\mathbb{R}$.

By (29) we have one of 2 cases:

1. $F_{D}^{+}$is a minimal conic bundle with 6 singular fibers. In this case $F_{D}^{+}(\mathbb{R}) \sim$ $\uplus 3 S^{2}$, a contradiction.

2. $F_{D}^{+}$is the blow up of a Del Pezzo surface of degree 3 or 4 over $\mathbb{R}$. By (89) $F_{D}^{+}(\mathbb{R})$ has at most 2 connected components, a contradiction.

$(7) \Rightarrow(1)$ : Assume that $D$ has a complex bitangent $L$. Its conjugate $\bar{L}$ is again a bitangent. Let $C \subset F_{D}^{+}$be a complex line over $L$. Its conjugate $\bar{C}$ lies over $\bar{L}$. Then $(C \cdot \bar{C}) \leq 1$ (the only possible intersection point lies over $L \cap \bar{L}$ ). Thus $F_{D}^{+}$has either a disjoint pair of conjugate lines or a conic bundle structure, a contradiction.

93 (Degree 1 Del Pezzo surfaces).

Let $F$ be a degree 1 Del Pezzo surface over any field $k .\left|-K_{F}\right|$ is a pencil with exactly one base point. So this is a $k$-point and $F(k) \neq \emptyset .\left|-2 K_{F}\right|$ is base point free and exhibits $F$ as a double cover of a quadric cone $Q \subset \mathbb{P}^{3}$, ramified along a curve $D \subset Q$ which is a complete intersection of $Q$ with a cubic surface with equation $(f=0)$. $D$ does not pass through the vertex of the cone.

$F_{\bar{k}}$ contains 240 lines (that is -1-curves); cf. [Manin72, IV.4.3]. We obtain these as follows. Take a plane $H \subset \mathbb{P}^{3}$ which is tangent to $D$ at 3 points. The preimage of $H \cap Q$ in $F$ has 2 irreducible components, each is a line. Thus we conclude that there are 120 planes which are tangent to $D$ at 3 points.

Assume now that $k=\mathbb{R}$. Since $Q(\mathbb{R}) \neq \emptyset$, we can write $Q=\left(x^{2}+y^{2}=1\right)$ in suitable affine coodinates $(x, y, z)$ on $\mathbb{A}^{3}$. That is, $Q(\mathbb{R})$ is a cylinder with a singular point at infinity.

As in the degree 2 case, for each (nonhomogeneous) cubic $f(x, y, z)$ we obtain two degree 1 Del Pezzo surfaces, given by affine equations

$$
F_{f}^{ \pm}:=\left(x^{2}+y^{2}-1=u^{2} \mp f(x, y, z)=0\right) \subset \mathbb{A}^{4} .
$$

There are 2 types of simple closed loops on a cylinder: null homotopic ones (I call them ovals) and those homotopic to a plane section (I call them big circles).

Since $D(\mathbb{R})$ is the intersection of a cylinder with a cubic, it has 3 or 1 intersection points with any ruling line of the cylinder. Thus $D(\mathbb{R})$ contains either 3 big circles (and no ovals) or 1 big circle. $D$ has genus 4 , hence by Harnack's theorem $(2), D(\mathbb{R})$ has at most 5 connected components. An oval can not be inside another oval since this would give 4 points on a ruling. Furthermore, we can not have an oval on one side the big circle and at least two ovals on the other side. Indeed, choosing points $P_{1}, P_{2}, P_{3}$ inside the 3 ovals, there is a plane $H$ through them. Then $H$ intersects 
each oval in at least 2 points, and also the big circle. So $(H \cdot D) \geq 8$, but $D$ has degree 6 , a contradiction.

If all the ovals are on the same side of the big circle, we can normalize $f$ so that it is positive on the other side. The other cases are symmetrical and it makes little sense to normalize $f$.

We can summarize these results in the following table:

PROPOSITION 94. There are 7 topological types of degree 6 smooth real complete intersection curves on the cylinder $\left(x^{2}+y^{2}=1\right)$, not passing through the point at infinity. Correspondingly there are 11 topological types of degree 1 real Del Pezzo surfaces. The following table gives the complete list. The types in the same row correspond to each other under $D=(f=0) \cap Q \leftrightarrow F_{f}^{+} \leftrightarrow F_{f}^{-}$.

\begin{tabular}{|c|c|c|}
\hline$D(\mathbb{R})$ & $F_{f}^{+}(\mathbb{R})$ & $F_{f}^{-}(\mathbb{R})$ \\
\hline 1 big circle +4 ovals & $\mathbb{R P}^{2} \uplus 4 S^{2}$ & $\# 9 \mathbb{R} \mathbb{P}^{2}$ \\
\hline 1 big circle +3 ovals & $\mathbb{R P}^{2} \uplus 3 S^{2}$ & $\# 7 \mathbb{R P}^{2}$ \\
\hline 1 big circle +2 ovals & $\mathbb{R P}^{2} \uplus 2 S^{2}$ & $\# 5 \mathbb{R} \mathbb{P}^{2}$ \\
\hline 1 big circle +1 oval & $\mathbb{R P}^{2} \uplus S^{2}$ & $\# 3 \mathbb{R}^{2}$ \\
\hline 1 big circle +0 oval & $\mathbb{R P}^{2}$ & $\mathbb{R P}^{2}$ \\
\hline 1 big circle $+1+1$ ovals & $\# 3 \mathbb{R P}^{2} \uplus S^{2}$ & $\# 3 \mathbb{R P}^{2} \uplus S^{2}$ \\
\hline 3 big circles & $\mathbb{R P}^{2} \uplus \# 2 \mathbb{R} \mathbb{P}^{2}$ & $\mathbb{R P}^{2} \uplus \# 2 \mathbb{R P}^{2}$ \\
\hline
\end{tabular}

The following theorem, due to [Comessatti13], is the degree 1 version of (92). I thank F. Russo for checking the arguments of Comessatti.

Theorem 95. Let $D=(f=0) \subset Q$ be a degree 6 smooth real complete intersection curve on the cylinder $Q=\left(x^{2}+y^{2}=1\right)$. The following are equivalent:

1. All 120 triple tangents of $D$ are real and $f$ is negative on all of them.

2. All 240 lines of $F_{f}^{-}$are real.

3. $F_{f}^{-}$is isomorphic to $\mathbb{P}_{\mathbb{R}}^{2}$ blown up in 8 real points.

4. $F_{f}^{-}(\mathbb{R}) \sim \# 9 \mathbb{R}^{2}$.

5. $D(\mathbb{R}) \sim \uplus 5 S^{1}$.

6. $F_{f}^{+}(\mathbb{R}) \sim \mathbb{R P}^{2} \uplus 4 S^{2}$.

7. $F_{f}^{+}$has Picard number 1 over $\mathbb{R}$.

Proof. (1) $\Rightarrow(2)$ : If $f$ is negative on a triple tangent then $u^{2}=-f$ has real solutions, giving a pair of real lines on $F_{f}^{-}$.

$(2) \Rightarrow(3)$ : Over $\mathbb{C}, F_{f}^{-}$is the blow up of $\mathbb{P}^{2}$ at 8 points. Thus it has 8 disjoint lines. If all lines are real, we have 8 disjont real lines. Contracting these we get a Del Pezzo surface of degree 9 over $\mathbb{R}$. By (29.D) it is $\mathbb{P}_{\mathbb{R}}^{2}$.

$(3) \Rightarrow(4)$ : Topologically, each blowing up is connected sum with $\mathbb{R}^{2}$.

$(4) \Rightarrow(5)$ : This follows from (94).

$(5) \Rightarrow(6)$ : This also follows from (94).

$(6) \Rightarrow(7)$ : Assume to the contrary that $F_{f}^{+}$has Picard number $\geq 2$ over $\mathbb{R}$. $F_{f}^{+}$can not be a minimal conic bundle since $\left(K^{2}\right)$ is odd. Thus $F_{f}^{+}$is the blow up of Del Pezzo surface of degree 2 or 3 over $\mathbb{R}$. By $(91,89) F_{f}^{+}(\mathbb{R})$ has at most 4 connected components, a contradiction.

$(7) \Rightarrow(1)$ : If $D$ has a complex triple tangent, we can argue as in $(92 .(7) \Rightarrow(1))$ that $F_{f}^{+}$contains a conjugate pair of lines $C, \bar{C}$ such that $(C \cdot \bar{C}) \leq 2 .(C+\bar{C}) \equiv r K$ for some $r \in \mathbb{Z}$, thus $2(C \cdot \bar{C})-2=r^{2}$. This is impossible. 
If there is a real triple tangent such that $f$ is positive on it then as in (1) $\Rightarrow$ (2) we get real lines on $F_{f}^{+}$.

Remark 96. It is not obvious that Del Pezzo surfaces with the same topological type form a connected family. Let us look for instance at cubics which are obtained from $\mathbb{P}^{2}$ by blowing up 6 real points. These correspond to 6 points in $\mathbb{P}^{2}$, no 3 on a line, not all on a conic. The possible configurations do not form a connected set.

On the other hand, a cubic surface like this is obtained as a blow up of $\mathbb{P}^{2}$ in many different ways, so the set of all such cubics still may be a connected set.

It is in fact true that all real Del Pezzo surfacs with the same degree and the same topological type form a connected family, with the exception of degree 8 surfaces without real points. For a modern proof see [DIK00, 17.3].

ACKnowledgments . I thank L. Bonavero, S. Cynk, S. Endrass, S. Kharlamov and W. Kucharz for numerous comments and improvements. A. Marin directed me to several 19th century references. F. Russo checked the arguments of Comessatti about degree 1 and 2 Del Pezzo surfaces and pointed out some misunderstandings on my part. Partial financial support was provided by the NSF under grant number DMS-0096268.

\section{References}

Akbulut-King91. S. Akbulut and H. King, Rational structures on 3-manifolds, Pacific J. Math. 150 (1991) 201-204

Artin68. M. Artin, The implicit function theorem in algebraic geometry, in: Algebraic geometry, Bombay, Oxford Univ. Press 1968, 13-34

BPV84. W. Barth, C. Peters and A. Van de Ven, Compact Complex Surfaces, Springer, 1984 Benedetti-Marin92. R. Benedetti and A. Marin, Déchirures de variétés de dimension trois, Comm. Math. Helv. 67 (1992) 514-545

BCR87. J. Bochnak, M. Coste and M-F. Roy, Géométrie algébrique réelle, Springer 1987; revised English translation: Real algebraic geometry, Springer, 1999

Bochnak-Kucharz99. J. Bochnak and W. Kucharz, The Weierstrass approximation theorem for maps between real algebraic varieties, Math. Ann. 314 (1999) 601-612.

Bouvier98. C. Bouvier, Diviseurs essentiels, composantes essentielles des variétés toriques singulières. Duke Math. J. 91 (1998) 609-620

Catanese-Frediani00. F. Catanese and P. Frediani, Real hyperelliptic surfaces and the orbifold fundamental group, preprint (2000)

Comessatti13. A. Comessatti, Fondamenti per la geometria sopra superfizie razionali dal punto di vista reale, Math. Ann. 73 (1913) 1-72

Comessatti14. A. Comessatti, Sulla connessione delle superfizie razionali reali, Annali di Math. 23(3) (1914) 215-283

DIK00. A. Degtyarev, I. Itenberg and V. Kharlamov, Real Enriques Surfaces, Lecture Notes in Mathematics no. 1746. Springer-Verlag, Berlin, 2000

Degtyarev-Kharlamov00. A. Degtyarev and V. Kharlamov, Topological properties of real algebraic varieties: Rokhlin's way, preprint

Degtyarev-Kharlamov01. A. Degtyarev and V. Kharlamov, Real rational surfaces are quasisimple, preprint

Harnack1876. Über die Vieltheiligkeit der ebenen algebraischen Kurven, Math. Ann. 10 (1876) 189-198

Hartshorne77. R. Hartshorne, Algebraic Geometry, Springer, 1977

Hayakawa99. T. Hayakawa, Blowing ups of 3-dimensional terminal singularities, Publ. Res. Inst. Math. Sci. 35 (1999) 515-570

Hempel76. J. Hempel, 3-manifolds, Princeton Univ. Press, 1976

Iskovskikh67. V. A. Iskovskikh, Rational surfaces with a pencil of rational curves, Math. USSR Sb. 3 (1967) 563-587 
Iskovskikh80. V. A. Iskovskikh, Minimal models of rational surfaces over arbitrary fields, Math. USSR Izv. 14 (1980) 17-39

Kawakita00. M. Kawakita, Divisorial contractions in dimension three which contract divisors to smooth points, (preprint) 2000

Kawamata92. Y. Kawamata, Boundedness of $\mathbb{Q}$-Fano threefolds, Proc. Int. Conf. Algebra, Contemp. Math. vol. 131 (1992) 439-445

Kharlamov78. V. M. Kharlamov, Real algebraic surfaces (Russian) Proceedings of the International Congress of Mathematicians (Helsinki, 1978) pp. 421-428, Acad. Sci. Fennica, Helsinki, 1980.

Kharlamov00. V. M. Kharlamov, Variétés de Fano réelles, Sém. Bourbaki, No. 872, March 2000

Klein1876. F. Klein, Über eine neuer Art von Riemannschen Flächen, Math. Ann. 10 (1876) 398416, Reprinted in : F. Klein, Gesammelte Mathematische Abhandlungen, Springer, 1922, vol. II.

Kollár91. J. Kollár, Flips, Flops, Minimal Models, etc., Surv. in Diff. Geom. 1 (1991) 113-199

Kollár96. J. Kollár, Rational Curves on Algebraic Varieties, Springer Verlag, Ergebnisse der Math. vol. 32, 1996

Kollár98a. J. Kollár, Low degree polynomial equations, in: European Congress of Math. Birkhäuser, 1998, 255-288

Kollár98b. J. Kollár, Real Algebraic Threefolds I. Terminal Singularities, Collectanea Math. (FERRAN SERRANO, 1957-1997) 49 (1998) 335-360

Kollár98c. J. Kollár, The Nash conjecture for algebraic threefolds, ERA of AMS 4 (1998) 63-73

Kollár99a. J. Kollár, Real Algebraic Threefolds II. Minimal Model Program, Jour. AMS 12 (1999) 33-83

Kollár99b. J. Kollár, Real Algebraic Threefolds III. Conic Bundles, J. Math. Sci. (New York) 94 (1999) 996-1020

Kollár00a. J. Kollár, Real Algebraic Threefolds IV. Del Pezzo Fibrations, in: Complex analysis and algebraic geometry, de Gruyter, Berlin, 2000, 317-346,

Kollár00b. J. Kollár, The nonprojective Nash conjecture (preprint)

Kollár-Mori98. J. Kollár and S. Mori, Birational geometry of algebraic varieties, English edition: Cambridge Univ. Press, 1998; Japanese edition: Iwanami Shoten, 1998

Kollár-Smith97. J. Kollár and K. Smith, Rational and Non-rational Algebraic Varieties (e-prints: alg-geom/9707013)

Manin66. Yu. I. Manin, Rational surfaces over perfect fields (in Russian), Publ. Math. IHES 30 (1966) 55-114

Manin72. Yu. I. Manin, Cubic forms (in Russian), Nauka, 1972. English translation: NorthHolland, 1974, second expanded edition, 1986

Markushevich96. D. G. Markushevich, Minimal discrepancy for a terminal cDV singularity is 1 , J. Math. Sci. Univ. Tokyo 3 (1996) 445-456

Mikhalkin97. G. Mikhalkin, Blow up equivalence of smooth closed manifolds, Topology, 36 (1997) 287-299

Milnor64. J. Milnor, On the Betti numbers of real varieties. Proc. Amer. Math. Soc. 15 (1964) 275-280

Milnor-Stasheff74. J. Milnor and J. Stasheff, Characteristic classes, Princeton Univ. Press, 1974

Moishezon67. B. Moishezon, On $n$-dimensional compact varieties with $n$ algebraically independent meromorphic functions, Amer. Math. Soc. Transl. 63 (1967) 51-177

Mori82. S. Mori, Threefolds whose Canonical Bundles are not Numerically Effective, Ann. of Math. 116 (1982) 133-176

Nash52. J. Nash, Real algebraic manifolds, Ann. Math. 56 (1952) 405-421

Nakano70. S. Nakano, On the inverse of monoidal transformations, Publ. Res. Inst. Math. Sci. Kyoto, 6 (1970) 483-503

Narasimhan68. R. Narasimhan, Analysis on real and complex manifolds, North-Holland, 1968

Nikulin79. V. V. Nikulin, Integer symmetric bilinear forms and some of their geometric applications. (Russian) Izv. Akad. Nauk SSSR Ser. Mat. 43 (1979) 111-177

Plücker1839. J. Plücker, Theorie der algebraischen Kurven, Bonn, 1839

Reid85. M. Reid, Young person's guide to canonical singularities, in Algebraic Geometry, Proc. Symp. Pure Math. vol.46, pp. 345-414

Rolfsen76. D. Rolfsen, Knots and links, Publish or Perish, 1976 
Schläfli1863. L. Schläfli, On the distribution of surfaces of the third order into species, Phil. Trans. Roy. Soc. London, 153(1863)193-241. Reprinted in : L. Schläfli, Gesammelte Mathematische Abhandlungen, Birkhäuser, 1953, vol. II.

Scott83. P. Scott, The geometries of 3-manifolds, Bull. London Math. Soc., 15 (1983) 401-487

Segre42. B. Segre, The non-singular cubic surfaces, Clarendon Press, 1942

Segre51. B. Segre, The rational solutions of homogeneous cubic equations in four variables, Notae Univ. Rosario 2 (1951) 1-68

Shafarevich72. R. I. Shafarevich, Basic Algebraic Geometry (in Russian), Nauka, 1972. English translation: Springer, 1977, second expanded edition, 1994

Silhol84. R. Silhol, Real algebraic surfaces with rational or elliptic fibering, Math. Zeitschr. 186(1984) 465-499

Silhol89. R. Silhol, Real algebraic surfaces, Springer Lecture Notes vol. 1392, 1989

Sullivan71. D. Sullivan, Combinatorial invariants of analytic spaces, Springer Lecture Notes vol. $192,(1971) 165-168$

Sun95. X. Sun, A regularity theorem on birational morphisms, J. Algebra 178 (1995) 919927

Thom65. R. Thom, Sur l'homologie des variétés algébriques réelles, in: Differential and combinatorial topology, Princeton Univ. Press, 1965, 255-265

Tognoli73. A. Tognoli, Su una congettura di Nash, Ann. Sci. Norm. Sup. Pisa 27 (1973) 167-185

Viro90. O. Ya. Viro, Real algebraic plane curves, Leningrad Math. J. 1 (1990) 1059-1134

Viterbo98. C. Viterbo, Symplectic real algebraic geometry, to appear

Zeuthen1874. H.G. Zeuthen, Sur les différentes formes des courbes du quatrième ordre, Math. Ann. 7(1874) 410-432

Princeton University, Princeton NJ 08544-1000

kollar@math.princeton.edu 
